\title{
Clustered Fine Compartmentalization of the Mouse Embryonic Cerebellar Cortex and Its Rearrangement into the Postnatal Striped Configuration
}

\author{
Hirofumi Fujita, ${ }^{1}$ Noriyuki Morita, ${ }^{2,3}$ Teiichi Furuichi, ${ }^{3,4}$ and Izumi Sugihara ${ }^{1}$ \\ ${ }^{1}$ Department of Systems Neurophysiology, Graduate School of Medical and Dental Sciences, Tokyo Medical and Dental University, 1-5-45 Yushima, \\ Bunkyo-ku, Tokyo 113-8519, Japan, 22Department of Nutritional Sciences, Faculty of Human Ecology, Yasuda Women's University, 6-13-1 Yasuhigashi, \\ Asaminami, Hiroshima 731-0153, Japan, ${ }^{3}$ Laboratory for Molecular Neurogenesis, RIKEN Brain Science Institute, 2-1 Hirosawa, Wako, Saitama 351-0198, \\ Japan, and ${ }^{4}$ Department of Applied Biological Science, Tokyo University of Science, Noda, Chiba 278-8510, Japan
}

Compartmentalization is essential for a brain area to be involved in different functions through topographic afferent and efferent connections that reflect this organization. The adult cerebellar cortex is compartmentalized into longitudinal stripes, in which Purkinje cells (PCs) have compartment-specific molecular expression profiles. How these compartments form during development is generally not understood. To investigate this process, we focused on the late developmental stages of the cerebellar compartmentalization that occur from embryonic day 17.5 (E17.5), when embryonic compartmentalization is evidently observed, to postnatal day 6 (P6), when adult-type compartmentalization begins to be established. The transformation between these compartmentalization patterns was analyzed by mapping expression patterns of several key molecular markers in serial cerebellar sections in the mouse. A complete set of 54 clustered PC subsets, which had different expression profiles of FoxP2, PLC $\beta 4$, EphA4, Pcdh10, and a reporter molecule of the 1NM13 transgenic mouse strain, were distinguished in three-dimensional space in the E17.5 cerebellum. Following individual PC subsets during development indicated that these subsets were rearranged from a clustered and multilayered configuration to a flattened, single-layered and striped configuration by means of transverse slide, longitudinal split, or transverse twist spatial transformations during development. The Purkinje cell-free spaces that exist between clusters at E17.5 become granule cell raphes that separate striped compartments at P6. The results indicate that the $\sim 50$ PC clusters of the embryonic cerebellum will ultimately become the longitudinal compartments of the adult cerebellum after undergoing various peri- and postnatal transformations that alter their relative spatial relationships.

\section{Introduction}

The adult cerebellar cortex is subdivided transversely by its lobular folding (Larsell, 1952) and longitudinally by compartments of Purkinje cell (PC) subsets that are defined by the expression patterns of certain molecules. For example, in adulthood, aldolase C (zebrin II) is highly expressed in PCs in 20 longitudinally arranged compartments (Brochu et al., 1990). Longitudinal compartments have specific projection patterns of efferent PC axons and afferent olivocerebellar axons (Voogd et al., 2003; Sugihara and Shinoda, 2004; Sugihara et al., 2009), and are involved in different aspects of movement control and other cerebellar functions (Horn et al., 2010).

Received April 5, 2012; revised Aug. 8, 2012; accepted Sept. 4, 2012.

Author contributions: H.F. and I.S. designed research; H.F. and I.S. performed research; H.F., N.M., T.F., and I.S. contributed unpublished reagents/analytic tools; H.F. and I.S. analyzed data; H.F. and I.S. wrote the paper.

This work was supported by the Grants-in-Aid for Scientific Research (23.5291 to H.F. and 20300137 to I.S.) from the Japan Society for the Promotion of Science (JSPS). H.F. is a research fellow of JSPS. We thank Mr. Toshinori Unno for genotyping 1NM13 mice, Dr. Kazuhiko Sawada for instructing whole-mount immunostaining technique, and Dr. Sascha du Lac for comments on the manuscript.

The authors declare no competing financial interests.

Correspondence should be addressed to Dr. Izumi Sugihara, Department of Systems Neurophysiology, Tokyo Medical and Dental University Graduate School, 1-5-45 Yushima, Bunkyo-ku, Tokyo 113-8519, Japan. E-mail: isugihara.phy1@tmd.ac.jp.

DOI:10.1523/JNEUROSCI.1710-12.2012

Copyright $\odot 2012$ the authors $\quad 0270-6474 / 12 / 3215688-16 \$ 15.00 / 0$
The cerebellar compartmentalization and compartmentspecific axonal projections are established during development. PCs arise at embryonic day 10.5 (E10.5)-E12.5 and migrate upward to form a multi-cell-thick immature PC layer at $\sim$ E14.5 (Goffinet, 1983). The immature PC layer is compartmentalized into several aggregations of PCs in the late embryonic period (Korneliussen, 1968; Altman and Bayer, 1997). Compartmentalization of the PC layer is also revealed in the area-dependent difference in the expression profiles of molecules during this period (Millen et al., 1995; Wilson et al., 2011). Since mice that lack one of these molecules failed to establish proper olivocerebellar (EphA4; Hashimoto et al., 2012) and spinocerebellar projections (En1 and En2; Sillitoe et al., 2010), embryonic molecular compartmentalization seems to be essential for the establishment of functional cerebellar organization.

The details of how the embryonic cerebellar compartments are organized and transformed to the adult compartments are generally unclear. The sets of embryonic compartments that have been reported (Wassef and Sotelo, 1984; Oberdick et al., 1993; Millen et al., 1995; Larouche et al., 2006; Wilson et al., 2011) have barely been consistent with each other. These studies did not look at the entire cerebellar cortex or fully identify the immature lobules in which the compartments were located. Additionally, these 
Table 1. Antibody specification

\begin{tabular}{|c|c|c|c|c|}
\hline & Antigen & Immunogen & $\begin{array}{l}\text { Manufacturer, species, mono- or polyclonal, catalog and } \\
\text { lot no }\end{array}$ & Dilution used \\
\hline \multirow[t]{8}{*}{ Primary antibodies } & Calbindin-D28k & $\begin{array}{l}\text { Calbindin-D28k purified from chicken gut was used as the } \\
\text { immunogen }\end{array}$ & $\begin{array}{l}\text { Sigma-Aldrich, mouse monoclonal, Cat. \# C-8666, Lot \# } \\
\text { 058H4812 }\end{array}$ & $1: 6000$ \\
\hline & Calbindin-D28k & $\begin{array}{l}\text { Synthetic peptide derived from amino acids } 185-199 \text { of human } \\
\text { Calbindin-D-28K }\end{array}$ & AnaSpec, rabbit polyclonal, Cat. \# 53283, Lot \# GL141 & 1:4000 \\
\hline & EphA4 & $\begin{array}{l}\text { Purified, NSO-derived, recombinant mouse EphA4 extracellular } \\
\text { domain }\end{array}$ & $\begin{array}{l}\text { R\&D Systems, goat polyclonal, Cat. \# AF641, Lot \# } \\
\text { BVX0308091 }\end{array}$ & $1: 1000$ \\
\hline & FoxP2 & $\begin{array}{l}\text { Synthetic peptide derived from C-terminal amino acids of } \\
\text { isoforms I, II, IV, and V of human FOXP2: REIEEEPLSEDLE }\end{array}$ & $\begin{array}{l}\text { Everest Biotech, goat polyclonal, Cat. \# EB05226, Lot \# } \\
\quad 160409\end{array}$ & $\begin{array}{l}\text { 1:5000 for fluorescent, } \\
\text { 1:10000 for DAB }\end{array}$ \\
\hline & FoxP2 & $\begin{array}{l}\text { A KLH-conjugated synthetic peptide between } 664 \text { and } 693 \\
\text { amino acids from the C-terminal region of human FOXP2 }\end{array}$ & $\begin{array}{l}\text { Abgent, rabbit polyclonal, Cat. \# AP5753b, Lot\# } \\
\text { SA100916AA }\end{array}$ & 1:1000 \\
\hline & Pcdh10 & $\begin{array}{l}\text { The cytoplasmic domain of the } 0 \mathrm{~L}-\mathrm{pc} \text { isoform (Aoki et al., } \\
\text { 2003) }\end{array}$ & $\begin{array}{l}\text { Millipore, rat monoclonal, clone 5G10, Cat. \# MABT20, } \\
\text { Lot \# NRG1759424 }\end{array}$ & $1: 1600$ \\
\hline & $\mathrm{PLC} \beta 4$ & Amino acids $876-1115$ of $P L C \beta 4$ of human origin & $\begin{array}{l}\text { Santa Cruz Biotechnology, rabbit polyclonal, Cat. \#sc- } \\
\text { 20760, Lot\# A1508 }\end{array}$ & 1:150 \\
\hline & $\operatorname{ROR} \alpha$ & $\begin{array}{l}\text { Amino acids 196-260 mapping within an internal region of } \\
\text { ROR } \alpha 2 \text { of human origin }\end{array}$ & $\begin{array}{l}\text { Santa Cruz Biotechnology, rabbit polyclonal, Cat. \# } \\
\text { sc-28612, Lot \# } 10909\end{array}$ & 1:100 \\
\hline \multirow[t]{7}{*}{ Secondary antibodies } & \multicolumn{2}{|c|}{ Anti-goat lgG, biotin-conjugated } & Vector Laboratories, rabbit, Cat. \# BA-5000 & $1: 333$ \\
\hline & \multicolumn{2}{|c|}{ Anti-rabbit lgG, biotin-conjugated } & Vector Laboratories, goat, Cat.\# BA-1000 & $1: 333$ \\
\hline & \multicolumn{2}{|c|}{ Anti-mouse lgG, biotin-conjugated } & Vector Laboratories, goat, Cat. \# BA-9200 & 1:333 \\
\hline & \multicolumn{2}{|c|}{ Anti-goat lgG, fluorescein-conjugated } & Jackson ImmunoResearch, donkey, Cat. \# 705-095-147 & $1: 333$ \\
\hline & \multicolumn{2}{|c|}{ Anti-goat lgG, DyLight 488-conjugated } & Jackson ImmunoResearch, donkey, Cat. \# 705-485-147 & 1:400 \\
\hline & \multicolumn{2}{|c|}{ Anti-rabbit lgG, Texas Red-conjugated } & Jackson ImmunoResearch, donkey, Cat. \# 711-075-152 & $1: 333$ \\
\hline & \multicolumn{2}{|c|}{ Anti-rat lgG, DyLight 594-conjugated } & Jackson ImmunoResearch, donkey, Cat. \# 712-515-153 & 1:333 \\
\hline
\end{tabular}

The data are based on manufacturer's data sheets and in some cases on communications with manufacturers.
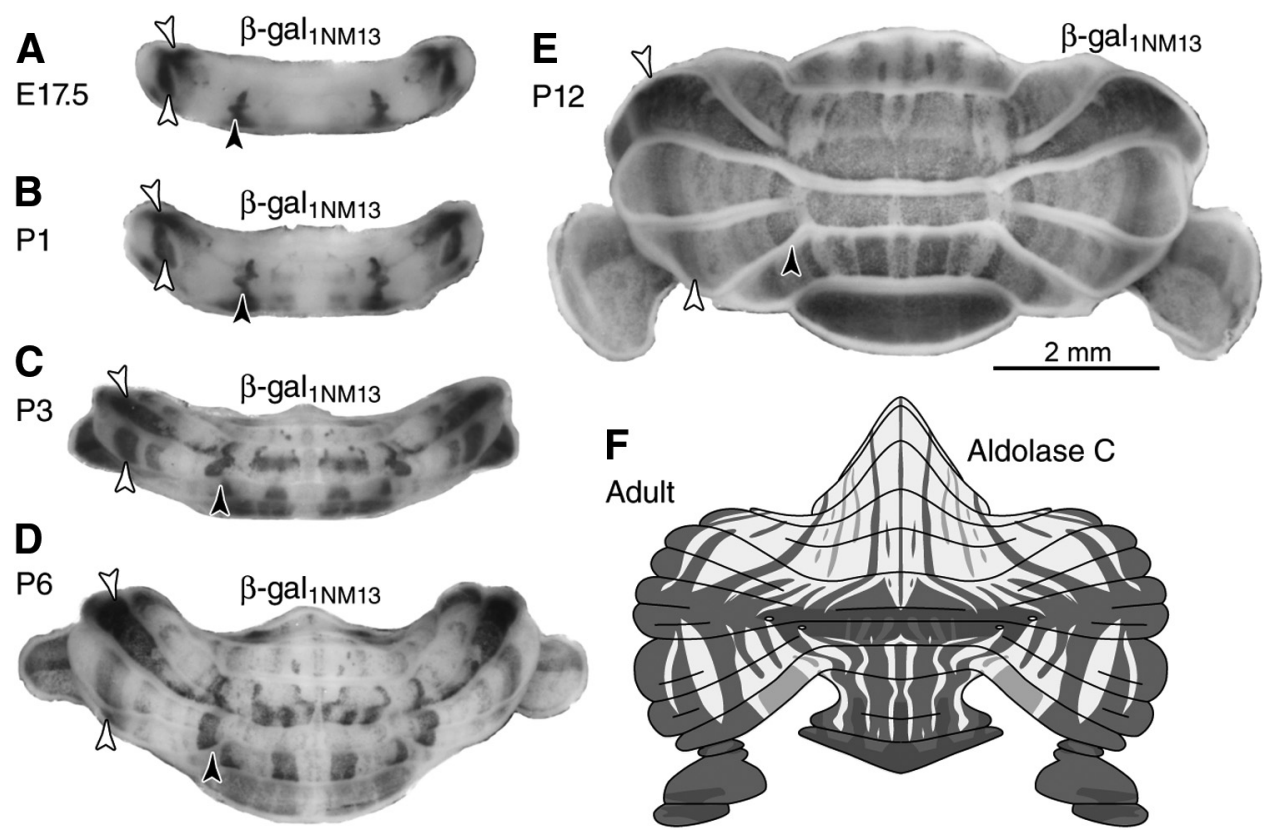

Figure 1. Development of the $\beta$-gal expression pattern in the 1NM13 transgenic mouse strain. $\boldsymbol{A}-\boldsymbol{E}$, Whole-mount $\beta$-gal visualization in the $1 \mathrm{NM} 13$ cerebellum at E17.5 (A), P1 (B), P3 (C), P6 $(\boldsymbol{D})$, and P12 (E). Dorsal view. Filled and open arrowheads indicate paramedian and hemispheral areas that begin to express $\beta$-gal earlier than other areas. These two areas can be clearly followed from E17.5 to P12, although many other $\beta$-gal-positive areas emerged between $\mathrm{P} 0$ and P12. Lobulation of the cerebellum also developed during this period. $\boldsymbol{F}$, Adult-type longitudinal compartments represented by the aldolase ( expression pattern mapped on the unfolded scheme of the entire cerebellar cortex (Sugihara and Quy, 2007). The $\beta$-gal expression pattern in the 1NM13 cerebellum at P12 is closely related to the aldolase C expression pattern (Furutama et al., 2010). Scale bar in $\boldsymbol{E}$ applies to $\boldsymbol{A}-\boldsymbol{E}$.

studies did not identify all of the clusters that comprise the entire population of PCs. Furthermore, compartment-dependent expression patterns of many molecules, including Pcp2 and Wnt7B (Smeyne et al., 1991; Millen et al., 1995), are not stable during development, which prevents a direct comparison between embryonic and adult compartments. Although birthdate-specific viral labeling and transgene-induced fate mapping have been used to trace some PC subsets through development (Hashimoto and
Mikoshiba, 2003; Sillitoe et al., 2009), embryonic compartmentalization has not yet been clarified for the entire cerebellar cortex.

Here, we labeled embryonic PCs for specific marker molecules (particularly FoxP2; Fujita and Sugihara, 2012) and carefully distinguished compartments by visualizing the combined expression profiles of several relatively stable molecules, including $\beta$-galactosidase in the $1 \mathrm{NM} 13$ transgenic mouse strain $(\beta$ - 


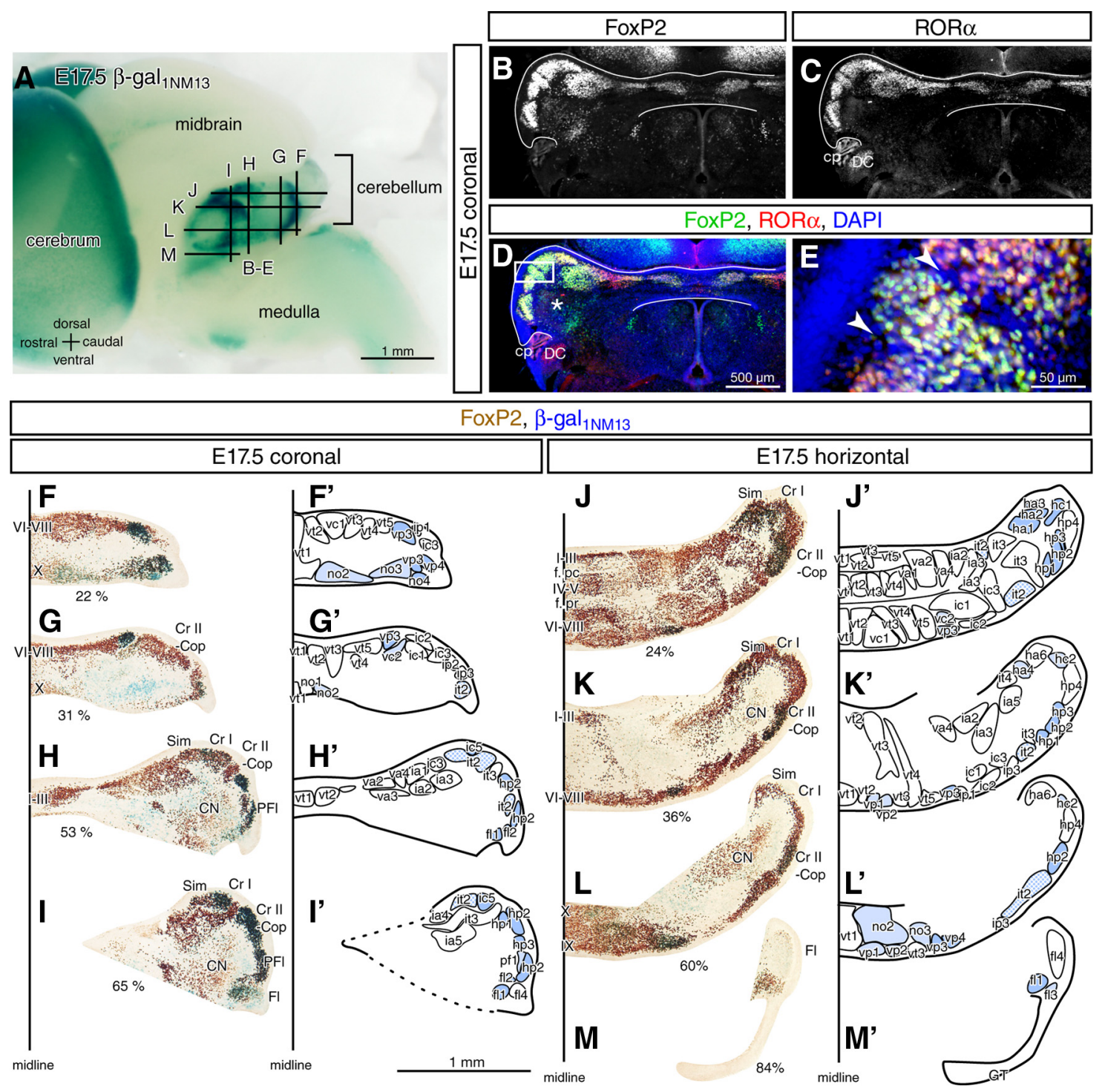

Figure 2. Compartmentalization into clusters in the E17.5PC layer. $A$, Lateral view of whole-mount $\beta$-gal ${ }_{1 \mathrm{NM} 13}$ visualization in the E17.5 brain. Lines indicate the level and direction of sections shown in $\boldsymbol{B}-\boldsymbol{M}$. $\boldsymbol{B}-\boldsymbol{E}$, Photomicrographs of immunostaining for PC-marker molecules, FoxP2 (B) and ROR $\alpha(\boldsymbol{C})$. These photos and DAPI counterstaining were merged into artificial color channels in a coronal section of the cerebellum at $E 17.5$ (D). Square in $\boldsymbol{D}$ indicates the area of the magnified photo in $\boldsymbol{E}$. Arrowheads in $\boldsymbol{E}$ indicate $\mathrm{PC}$-sparse space between $\mathrm{PC}$ clusters, which were filled with cells that were labeled with DAPI. $\boldsymbol{F}-\boldsymbol{M}^{\prime}$, Double-staining for $\beta$-gal ${ }_{1 \mathrm{NM} 13}$ (blue) and FoxP2 (brown) in coronal $(\boldsymbol{F}-\boldsymbol{I})$ and horizontal $(\boldsymbol{J}-\boldsymbol{M})$ sections. Note that FoxP2 expression is variable in intensity among $\mathrm{PC}$ clusters. Accompanying illustrations $\left(\boldsymbol{F}^{\prime}-\boldsymbol{M}^{\prime}\right)$ depict outlines of the $\mathrm{PC}$ clusters in the section with temporary names (see Results). Bluish colors in some depicted clusters in $\boldsymbol{F}^{\prime}-\boldsymbol{M}^{\prime}$ indicate the general expression intensity of $\beta$-gal ${ }_{1 \mathrm{NM} 13}$ in the clusters. Areas outside of the cerebellum have been trimmed. The relative position of individual sections within the entire caudorostral or dorsoventral range of the cerebellum is indicated as percentage in $\boldsymbol{F}-\boldsymbol{M}^{\prime}$. Scale bar in $\boldsymbol{D}$ applies to $\boldsymbol{B} \boldsymbol{- D}$; scale bar in $\boldsymbol{I}^{\prime}$ applies to $\boldsymbol{F}-\boldsymbol{M}^{\prime}$. Abbreviations in this and subsequent figures are as follows: I-X, Lobules I-X; a-c, sublobules a- C (as in Vla); C, caudal; CN, cerebellar nuclei; Cop, copula pyramidis; $\mathrm{cp}$, choroid plexus; $\mathrm{Cr}$ I, crus I of the ansiform lobule; $C \mathrm{rll}$, crus II of the ansiform lobule; $\mathrm{D}$, dorsal; $D C$, dorsal cochlear nucleus; $F l$, flocculus; f. ic, intercrural fissure; f. pc, preculminate fissure; f. pl, posterolateral fissure; f. pr, primary fissure; f. sec, secondary fissure; GT, germinal trigone; $L$, lateral; M, medial; Par, paramedian lobule; PFl, paraflocculus; R, rostral; Sim, simple lobule; V, ventral.

gal $_{1 \mathrm{NM} 13}$; Furutama et al., 2010), PLC $\beta 4$ (Marzban et al., 2007), EphA4 (Hashimoto and Mikoshiba, 2003), and Pcdh10 (Hirano et al., 1999). First, we identified and three-dimensionally reconstructed what appears to be the complete set of compartments that make up the complete PC population from serial sections of the E17.5 mouse cerebellum. Next, we followed these compartments from the embryonic to the postnatal cerebellum to reveal how the embryonic compartmentalization is transformed during development.

\section{Materials and Methods}

Animals. The experimental protocols described in this paper were approved by the Institutional Animal Care and Use Committee of Tokyo Medical and Dental University (100147, 0110302A, and 120093B). Em- bryos and pups [E17.5, $n=29$; postnatal day $0(\mathrm{P} 0), n=7 ; \mathrm{P} 1, n=12 ; \mathrm{P} 3$, $n=4 ; \mathrm{P} 6, n=17 ; \mathrm{P} 12, n=1]$ of the transgenic mouse line $1 \mathrm{NM} 13$ (Furutama et al., 1996, 2010) were used. This mouse strain carries a transgene that codes Escherichia coli $\beta$-galactosidase ( $\beta$-gal) protein fused to the nuclear localization signal that is driven by the IP3R1 gene promoter. This transgenic mouse strain was maintained on a $\mathrm{B} 6 \mathrm{C} 3 \mathrm{~F} 1$ genetic background by mating heterozygotic males with wild-type females that were supplied by a local breeder (CLEA Japan). $\beta$-Galactosidase expressed in heterozygotic $1 \mathrm{NM} 13$ mice is designated " $\beta$-gal ${ }_{1 \mathrm{NM} 13}$ " in this paper.

Histology. The day when the vaginal plug was detected in mating was designated E0.5. The day when the pups were born was considered P0. Anesthesia, perfusion, and fixation were performed as described previously (Fujita and Sugihara, 2012). To determine the genotypes (hetero- 

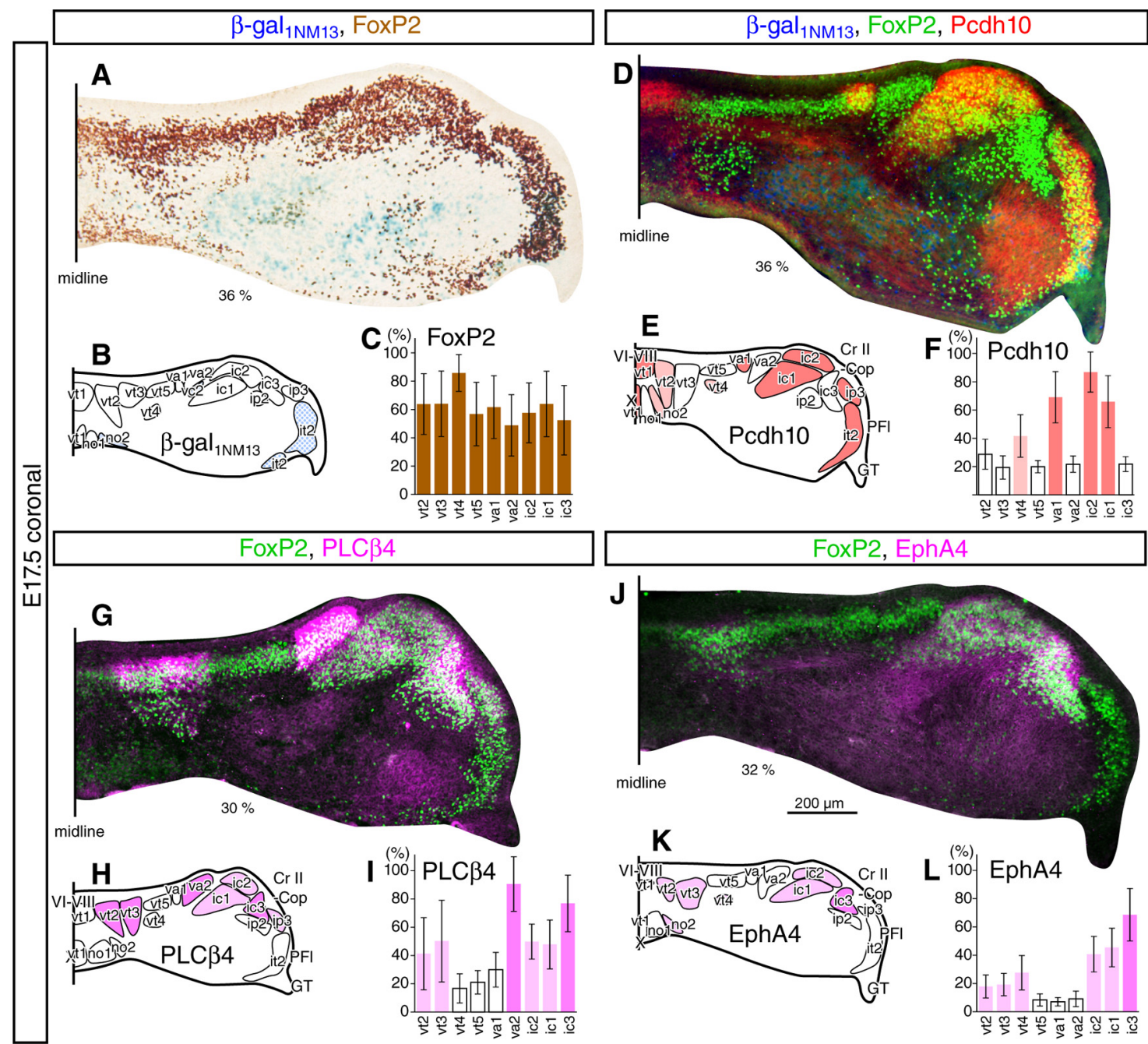

Figure 3. Distinction of clusters according to molecular expression profiles in the E17.5 PC layer. $A-L$, Double- or triple-staining for markers of $\mathrm{PC}$ clusters and FoxP2 accompanied by drawings of

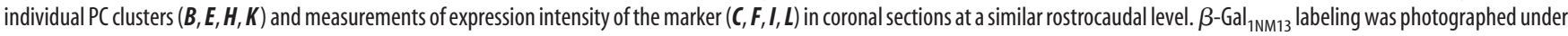
bright field (see Materials and Methods). Scale bar in $J$ applies to $A, D, G, J$.

zygote or wild-type) of the individual brains, the rostral part of the cerebrum was dissected and subjected to 5-bromo-4-chloro-3-indolyl$\beta$-D-galactopyranoside ( $\mathrm{X}$-gal) staining for $\beta$-gal visualization. $\beta$-Gal is highly expressed in the olfactory and other cerebral cortices in the heterozygote of the 1NM13 strain (Furutama et al., 1996). After being washed in PBS, the rostral cerebrum was incubated in $0.5 \mathrm{mg} / \mathrm{ml} \mathrm{X-gal}$ (Takara Shuzo), $3 \mathrm{~mm}$ potassium ferricyanide, $3 \mathrm{~mm}$ potassium ferrocyanide, $1 \mathrm{~mm} \mathrm{MgCl}_{2}$ in PBS (pH 7.4) plus $0.15 \%$ Triton $\mathrm{X}-100$ at $37^{\circ} \mathrm{C}$ overnight, and then rinsed several times in PBS. Indigo coloring indicated heterozygotes.

To visualize $\beta$-gal expression in the whole-mount preparation, the whole brain or the dissected cerebellum of heterozygotes was stained with X-gal (above) at $37^{\circ} \mathrm{C}$ for $30 \mathrm{~min}$ to $48 \mathrm{~h}$. For whole-cerebellum preparation, the cerebellum was dissected out from the rest of the brain before X-gal staining.

To make serial-section preparations, brains were coated with gelatin solution ( $10 \%$ gelatin, $10 \%$ sucrose in $10 \mathrm{~mm}$ phosphate buffer, $32^{\circ} \mathrm{C}$ ). The gelatin block was hardened by chilling and then soaked for 2 nights in fixative with a high sucrose content (5\% paraformaldehyde, $30 \%$ sucrose in 0.05 м phosphate buffer, $\mathrm{pH}$ 7.4). Coronal, horizontal, or sagittal sections were cut on a freezing microtome at a thickness of $40 \mu \mathrm{m}$, and a complete set of serial sections was collected. The ventral surface of the medulla at the level of the inferior olive was regarded as the horizontal plane.

To visualize $\beta$-gal expression in serial-section preparations, sections were stained with X-gal (above) before immunohistochemistry.
Immunohistochemistry with bright-field diaminobenzidine (DAB) visualization and fluorescent visualization were performed as described previously (Fujita and Sugihara, 2012). Goat anti-EphA4 (R\&D Systems), goat anti-FoxP2 (Everest Biotech), mouse anti-Calbindin-D28k (Sigma-Aldrich), rabbit anti-Calbindin-D28k (AnaSpec), rabbit antiFoxP2 (Abgent), rabbit anti-PLC $\beta 4$ (Santa Cruz Biotechnology), rabbit anti-ROR $\alpha$ (Santa Cruz Biotechnology), and rat anti-OL-protocadherin (Millipore) antibodies were used as primary antibodies. After being incubated with a mixture of appropriate primary antibodies, specimens were incubated with a mixture of appropriate secondary antibodies that had biotin or fluorescent tags. Table 1 contains detailed information on the antibodies used. The biotin-tagged secondary antibodies were then visualized with DAB. After immunohistochemistry, some sections were fluorescently counterstained with 4',6-diamidino-2-phenylindole, dihydrochloride (DAPI; -cellstain-DAPI, D212, Dojindo).

Whole-mount immunostaining was performed according to Sillitoe and Hawkes (2002), except that we used biotin-conjugated secondary antibody and biotinylated peroxidase-avidin complex instead of peroxidase-conjugated secondary antibody before DAB visualization, similar to the method for sections (above).

Bright-field sections were photographed using a film scanner (Dimage AF-5000, Minolta) at a resolution of 4800 pixels per inch for routine systematic analysis. Some sections were photographed using a digital camera (DP-50, Olympus) attached to a microscope (BX41, Olympus) for detailed analysis and for paper figures. Fluorescence sections were photographed with an appropriate filter in 16-bit gray- 

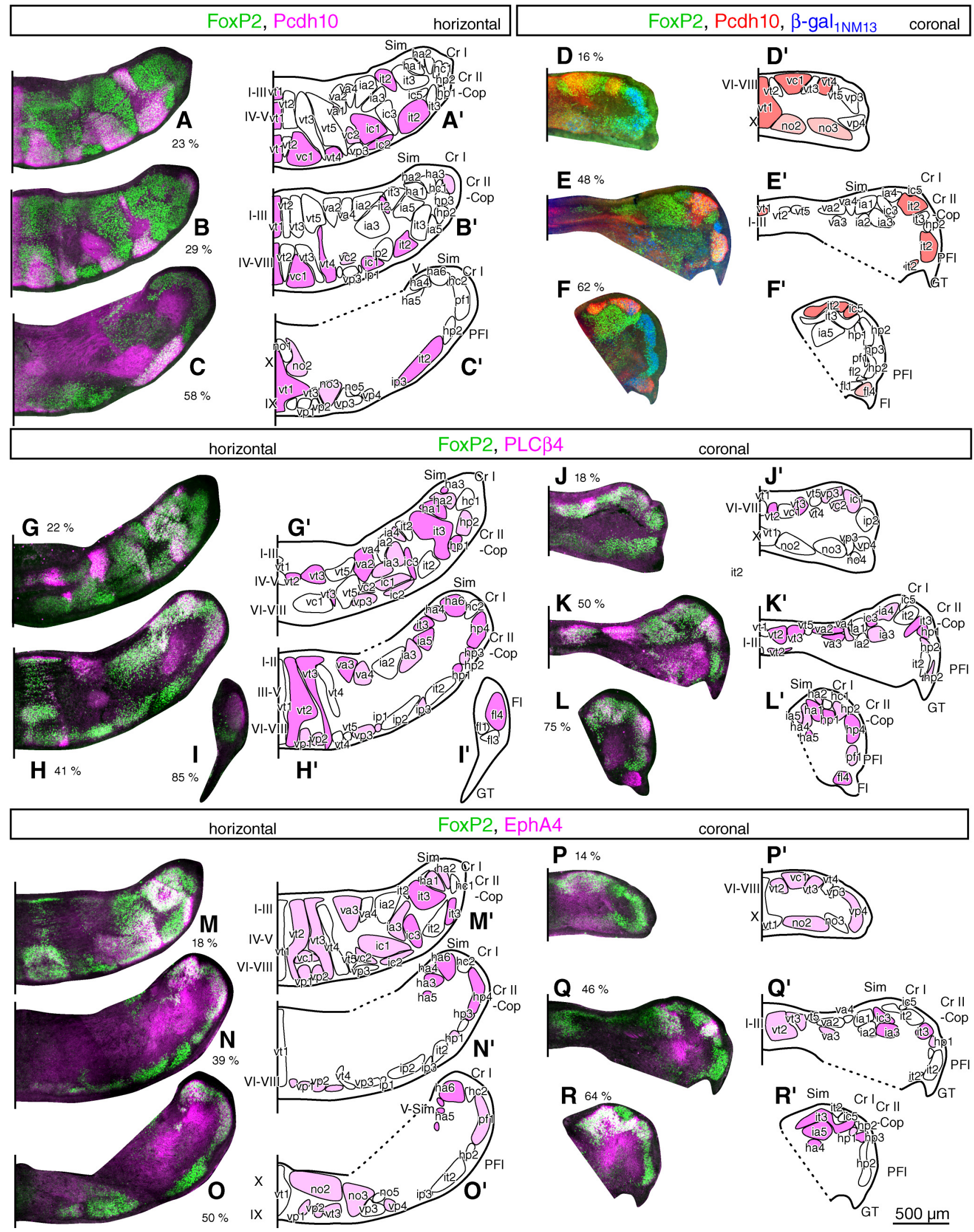

Figure 4. Expression of early cerebellar markers in E17.5 cerebellum at various levels of coronal and horizontal sections. $\boldsymbol{A}-\boldsymbol{R}^{\prime}$, Double-immunostaining for each of the early markers (red or magenta, $\boldsymbol{A}-\boldsymbol{F} ; \mathrm{Pcdh} 10 ; \boldsymbol{G}-\boldsymbol{L}, \mathrm{PLC} \boldsymbol{\beta} 4 ; \boldsymbol{M}-\boldsymbol{R}$, EphA4) and FoxP2 (green) accompanied by a drawing of individual PC clusters in the section $\left(\boldsymbol{A}^{\prime}-\boldsymbol{R}^{\prime}\right)$. $\boldsymbol{\beta}$-gal ${ }_{1 \mathrm{NM} 13}$ is also visualized in $\boldsymbol{D}-\boldsymbol{F}$. The drawings can be regarded as an atlas of the $P($ clusters (with our temporary names) at a particular level of the coronal or horizontal section. The magenta-like colors in drawings of some clusters indicate the intensity of expression of the marker in the section. Areas outside of the cerebellum are trimmed. The relative position (\%) of individual sections from dorsal to ventral or from caudal to rostral is indicated. The sectioning plane for $\boldsymbol{M} \mathbf{- 0}$ was slightly tilted toward the rostroventral-caudodorsal direction compared with other horizontal sections. Scale bar in $\boldsymbol{R}^{\prime}$ applies to $\boldsymbol{A}-\boldsymbol{R}^{\prime}$. 


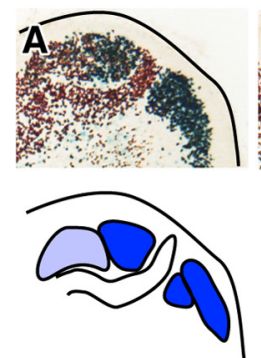

D

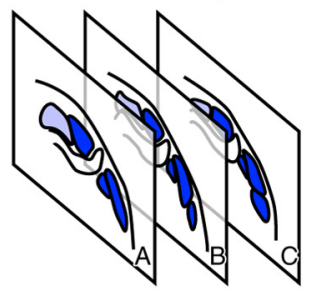

E
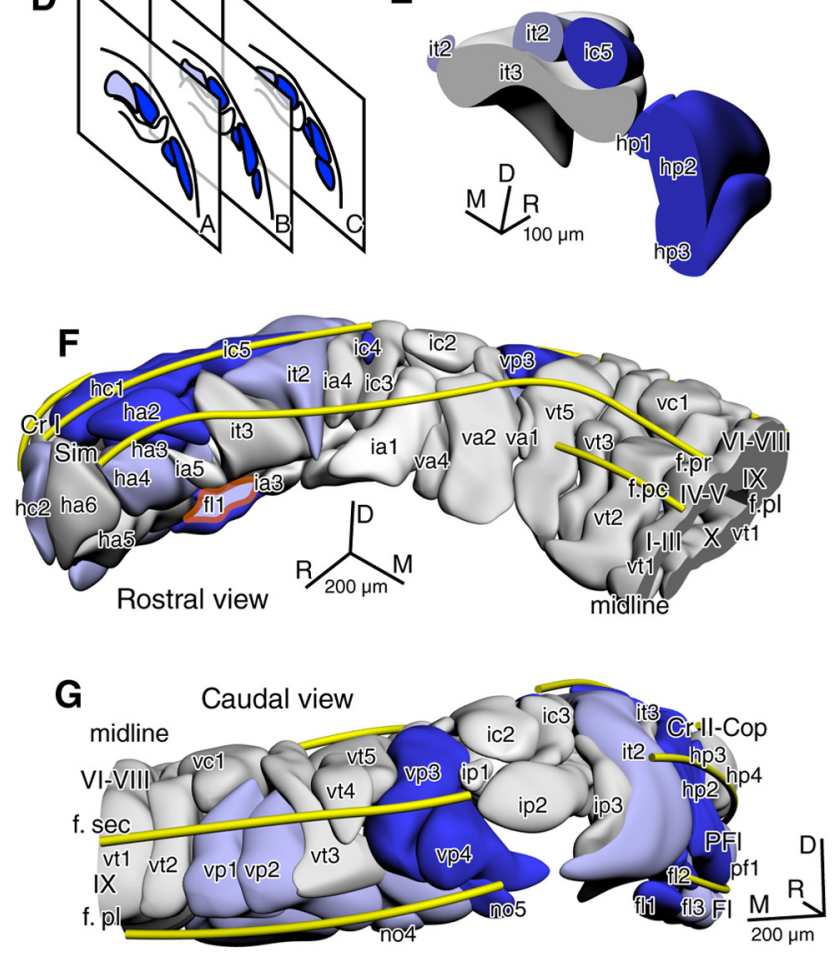
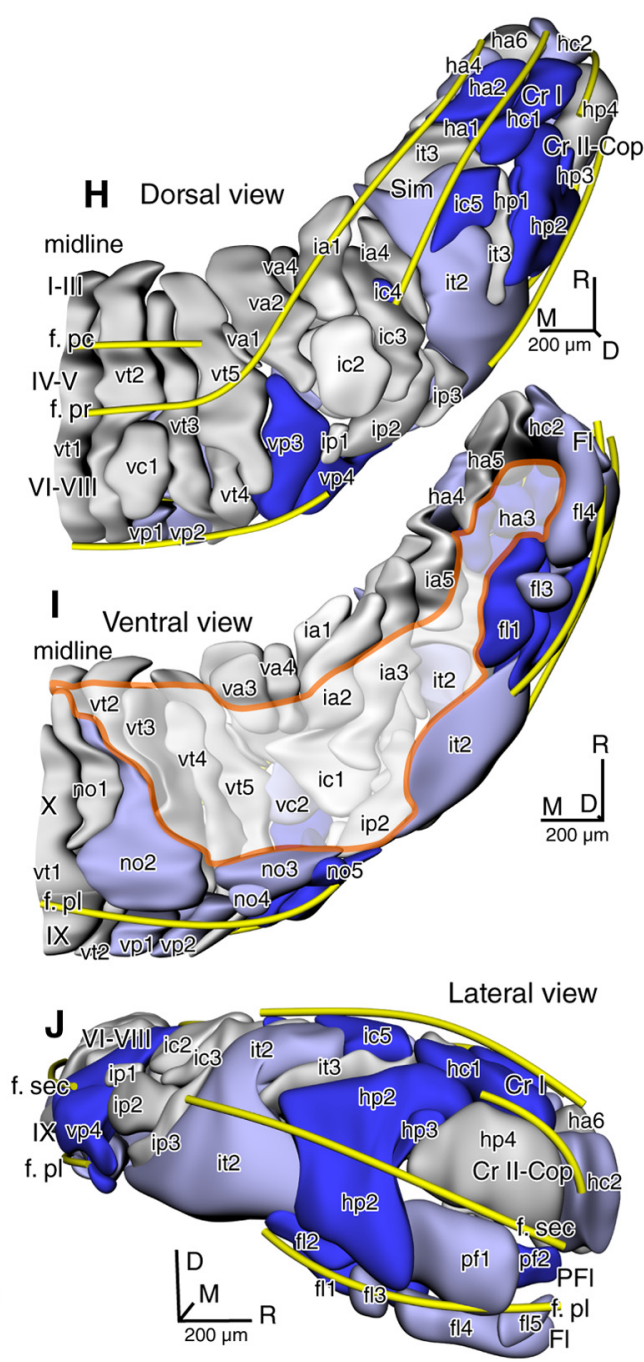

Figure 5. 3D reconstruction of all $E 17.5 \mathrm{PC}$ clusters. $\boldsymbol{A}-\boldsymbol{E}$, Methods for the $3 \mathrm{D}$ reconstruction of $\mathrm{PC}$ clusters. Contours of individual clusters depicted in consecutive coronal sections of the cerebellum $(\boldsymbol{A}-\boldsymbol{C})$ were aligned in 3D space (D). PC clusters were then reconstructed by using the "loft" command in Rhinoceros software $(\boldsymbol{E})$. $\boldsymbol{F}-\boldsymbol{J}$, Reconstruction of all PC clusters in the E17.5 hemicerebellum

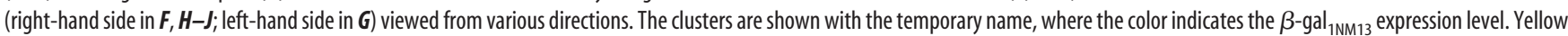
bars indicate cerebellar fissures that were recognized at E17.5. Opaque whitish areas circumscribed by an orange line indicate the inner part of the cerebellum (or clusters viewed through it). Scale bar in $\mathbf{C}$ applies to $\boldsymbol{A}-\boldsymbol{C}$.

scale using a cooled color CCD camera (DP-70, Olympus) attached to a conventional fluorescent microscope (BX51WI, Olympus). Wholemount specimens were photographed using a DP-70 camera attached to a macrozoom microscope (MVX10, Olympus). Photomicrographs were adjusted with regard to contrast and brightness and assembled using software (Photoshop 7, Adobe). An appropriate combination of pseudo-color was applied to the fluorescent photomicrographs using Photoshop. To match fluorescent and bright-field images of the same section for multilabeling, the bright-field photomicrograph taken in grayscale was digitally inverted in the intensity scale and then given pseudo-fluorescent color. In some figures, montages were made by combining two photomicrographs.

Identification of PC clusters. PC clusters were identified by the combined observation of PC layer arrangements, particularly PC gaps, and expression patterns of FoxP2, $\beta$-gal INM13 $_{1}$, PLC $\beta 4$, EphA4, and Pcdh10. Based on comparisons of four sets of multilabeled serial sections (FoxP2$\beta$-gal ${ }_{1 \mathrm{NM} 13}$, FoxP2- $\beta$-gal ${ }_{1 \mathrm{NM13}}$-Pcdh10, FoxP2-EphA4, and FoxP2PLC $\beta 4$ ), aggregations of PCs that had similar molecular expression profiles were identified.

Measurement of labeling intensity. The intensity of marker expression in a cluster (see Fig. 3C, F, I,L) was measured inside the cluster outline in a digital photomicrograph of a particular marker molecule using the 'select-circumscribe' and 'measure pixel brightness' tools in Photoshop. The average and SD of the pixel brightness $(0-100 \%)$ was calculated and displayed in a histogram with Excel (Microsoft).

Three-dimensional reconstruction of PC clusters from serial sections. When making drawings of identified clusters in serial sections, we superimposed drawings on photographs of coronal serial sections of the cerebellum that had been double-labeled for Foxp2 and $\beta$-gal ${ }_{1 \mathrm{NM} 13}$ as an accurate reference of position in Adobe Illustrator. The drawings were imported into three-dimensional (3D) graphics software (Rhinoceros 4, Robert McNeel \& Associates) and then sifted in the direction of the $z$-axis (rostrocaudal). The 3D model (see Model 1) was then reconstructed from drawings by using the "loft" command in Rhinoceros (Sugihara and Shinoda, 2007). Reconstructed PC clusters were colored in dark or light blue according to the $\beta$-gal INM13 $_{13}$ expression pattern.

Since accurate identification of clusters was essential in finalizing the $3 \mathrm{D}$ reconstruction, we carefully compared the cluster arrangement in the $3 \mathrm{D}$ reconstruction with the cluster arrangements in serial coronal and horizontal sections of other cerebella of the same developmental date. When discrepancy was observed, we revised cluster identification and reconstruction until no discrepancy was seen.

Serial section alignment analysis. To clearly distinguish between neighboring compartments, we clipped the same target area (a narrow trans- 
verse band of the PC layer) in photomicrographs of consecutive sections taken with either a AF-5000 film scanner or a DP-50 digital camera, and aligned the clipped areas in order. Some clipped areas were rotated and/or shifted so that they would show good alignment with neighboring sections.

Mapping of molecular expression patterns on the unfolded cerebellar cortex. To make schematic illustrations of the unfolded cerebellar cortices of mice at E17.5 and P6, the longitudinal (rostrocaudal) length of the PC layer of each lobule was measured in multiple Nissl-stained parasagittal sections every $200 \mu \mathrm{m}$ from the midsagittal plane. Since PCs were organized into clusters that sometimes overlay one another at E17.5 (see Results), the rostrocaudal length was measured at the most superficial positions of PC clusters in each combined lobule (lobules I-III, IV-V and so on) in Nissl-stained serial sagittal sections. For the paraflocculus and flocculus at P6, the cortical orientation of which was different from that of the rest of the cerebellum as in adults (see Results), the longitudinal length of the PC layer of each lobule was measured in coronal sections. The mediolateral length of the PC layer of each lobule was measured along the apex of each lobule. An unfolded scheme of the E17.5 and P6 mouse cerebellar cortex was then formulated based on these measured lengths by referring to a similar scheme the adult rat (Sugihara and Shinoda, 2004), adult mouse (Sugihara and Quy, 2007) and adult marmoset (Fujita et al., 2010). The 3D reconstruction of the E17.5 cerebellum (see Results) was also referred to when we made the E17.5 scheme. Crus I was arbitrarily made straight in the lateral direction for consistency in the unfolded schemes.

Molecular expression patterns visualized by X-gal staining or by immunostaining in serial coronal and horizontal sections were carefully mapped on the unfolded scheme in the same way as aldolase $\mathrm{C}$ and FoxP2 expression patterns were mapped in the unfolded schemes (Sugihara and Quy, 2007; Fujita and Sugihara, 2012). The mapped patterns were confirmed by serial section alignment analyses.

\section{Results}

\section{PC distribution in the E17.5 cerebellum}

To analyze compartmentalization of the PC layer in the embryonic cerebellum, the distribution of all PC populations had to be clarified. Since PCs were not clearly distinguishable from other cells morphologically at the embryonic stage, a specific marker molecule for embryonic PCs, such as FoxP2, was useful for identifying them. Double-labeling of FoxP2 and $\operatorname{ROR} \alpha$, another marker molecule for the nucleus of early PCs (Nakagawa et al., 1997), in E17.5 serial cerebellar sections showed that their labeling patterns coincided with each other in the PC layer throughout the cerebellar cortex (see Fig. $2 B-E$ ). Cells that were located relatively sparsely in the deep part of the cerebellum were labeled for FoxP2, but not for ROR $\alpha$ (see Fig. $2 D$, asterisk), and were hence regarded as nuclear neurons (Fujita and Sugihara, 2012). Close observation showed that PCs in a few areas in the flocculonodular lobe were scarcely labeled for FoxP2, as reported previously (Fujita and Sugihara, 2012), but were uniformly labeled for ROR $\alpha$ (data not shown). Thus, FoxP2 was regarded as a specific marker of embryonic PCs in most, but not all, lobules of the cerebellum. However, the labeling intensity of FoxP2 was much more variable among different areas of the embryonic PC layer than that of ROR $\alpha$ (see Fig. 2B,C). Since this was useful for identifying PC subsets (below), we preferentially used FoxP2 labeling in the present study.

PCs that were labeled for FoxP2 and/or ROR $\alpha$ formed aggregations in the immature PC layer (see Fig. $2 B-D$ ). As described in later sections, we designate the smallest aggregations of PCs, which were identified by their distinct molecular expression profiles, as "clusters" in this paper. Therefore, we cannot exclude the possibility that a cluster shown in the present study may be further subdivided. Individual clusters were often separated by space (designated "PC gaps") that was filled mainly with cells that were not labeled for

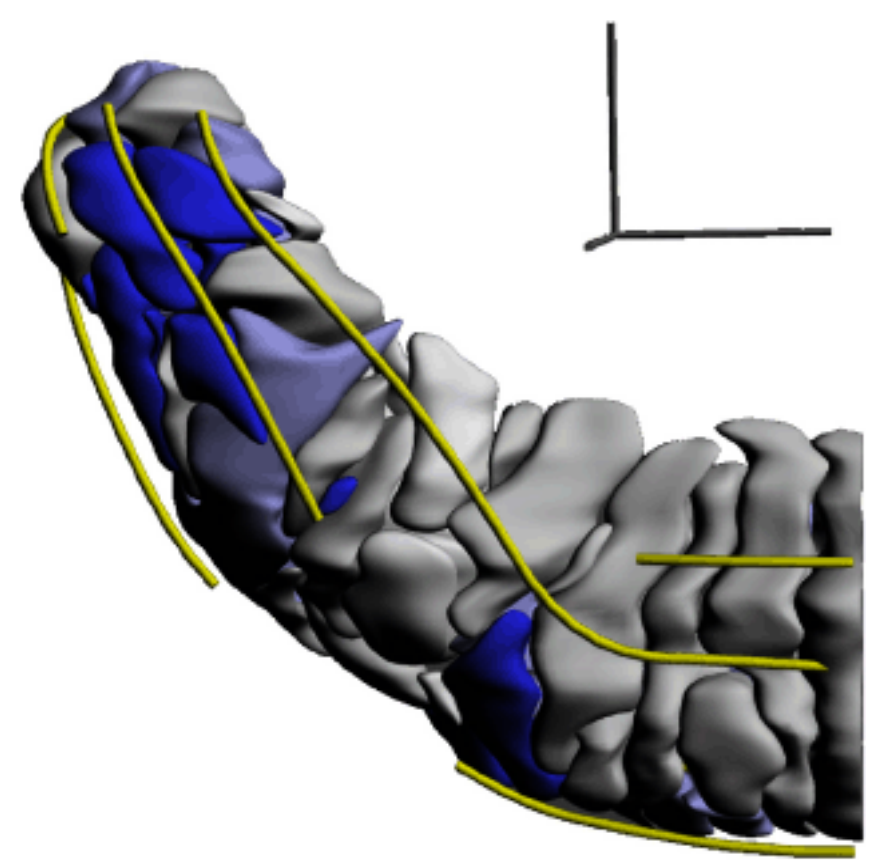

Model 1. 3D representation of E17.5PC clusters. All PC clusters and immature fissures in the E17.5 hemicerebellum (left-hand side) are represented in the 3D space. The contents are the same as shown in Figures $5 F-J$ and $8 B$. This file contains a list of 3D objects to show or hide individual clusters and fissures. They can also be viewed from any directions. "Left," "Top," "Front," "Right," "Bottom," "Back" views (in the view list of the file) actually shows the left (lateral), caudal, ventral, right (medial), rostral, dorsal aspects of the representation. Cluster vt1 and its midline cross section ("midline") are formed with multiple objects for technical reason in this $3 \mathrm{D}$ representation. Scale bars, $500 \mu \mathrm{m}$.

FoxP2 or ROR $\alpha$ (see Fig. $2 E$, arrowheads). Some PC gaps were wide enough to be consistently observed through serial sections.

\section{Identification of PC clusters based on expression patterns of marker molecules}

To identify clusters of the E17.5 PC layer, we looked at labeling patterns of several molecular markers of PC subsets as well as PC gaps. $\beta-\mathrm{Gal}_{1 \mathrm{NM} 13}$ is expressed in multiple stripe-shaped subsets (Furutama et al., 2010). Double-staining of FoxP2 (brown) and $\beta$-gal 1NM13 $_{13}$ (blue) in serial coronal and horizontal sections (see Fig. 2F-M) enabled us to identify PC clusters throughout the E17.5 PC layer by looking at PC gaps and expression patterns (presence or absence of expression and expression intensity) of $\beta$-gal ${ }_{1 \mathrm{NM} 13}$ and FoxP2. In the whole-mount preparation, $\beta$ - gal $_{1 \mathrm{NM} 13}$ is intensely expressed in two areas (medial and lateral) and weakly expressed in several neighboring areas (Figs. $1 A, 2 A$ ). In serial coronal and horizontal sections, these areas were clearly identified as distinct clusters and the positional relationship with regard to other neighboring clusters was mapped (Fig. $2 F^{\prime}-M^{\prime}$ ). For example, the medial intensely $\beta$-gal ${ }_{1 \mathrm{NM} 13}$-positive area was identified as clusters vp 3 and vp 4 (meaning \#3 and 4 in the vermis-posterior; see Results, below, for the nomenclature of clusters) and located in lobules VI-VIII, IX and X. The lateral intensely $\beta$-gal ${ }_{1 \mathrm{NM} 13}$-positive area was identified as clusters ha2, hc1 and hp2 and located in the simple lobule, crus I and crus II-copula pyramidis, respectively (Fig. $2 F-M^{\prime}$, blue clusters). Although many clusters were distinguished by double-labeling for FoxP2 and $\beta$-gal ${ }_{1 \mathrm{NM} 13}, \beta$-gal INM13 $_{13}$-negative PC clusters were not clearly identified with this double-labeling. Therefore, we examined the expression patterns of other specific marker molecules for PC subsets: PLC $\beta 4$, EphA4 and Pcdh10. Both heterozygous 

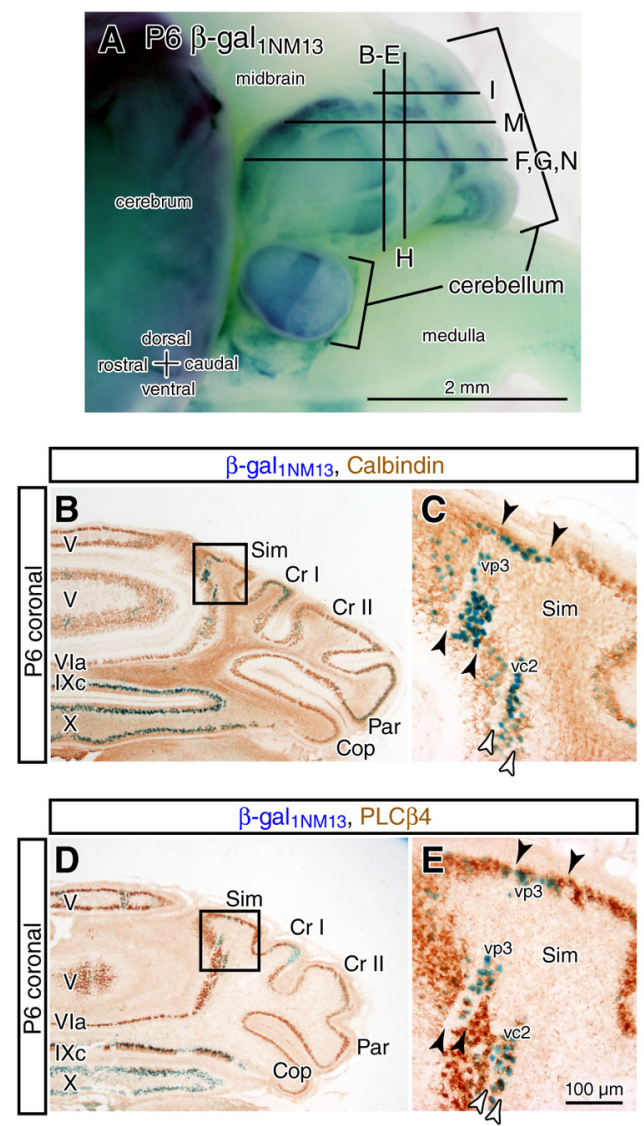
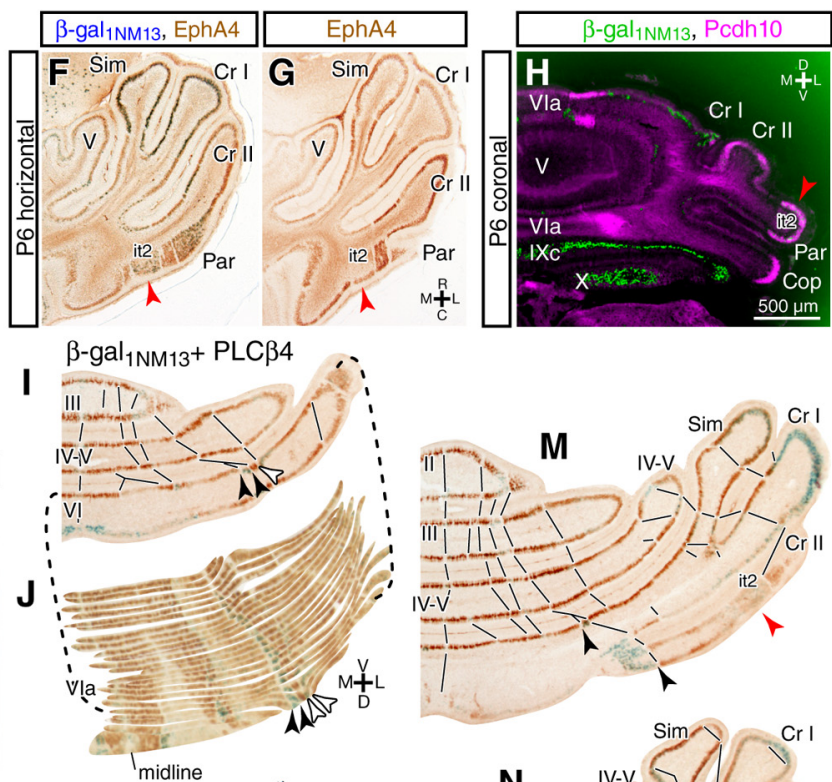

$\mathbf{K}$
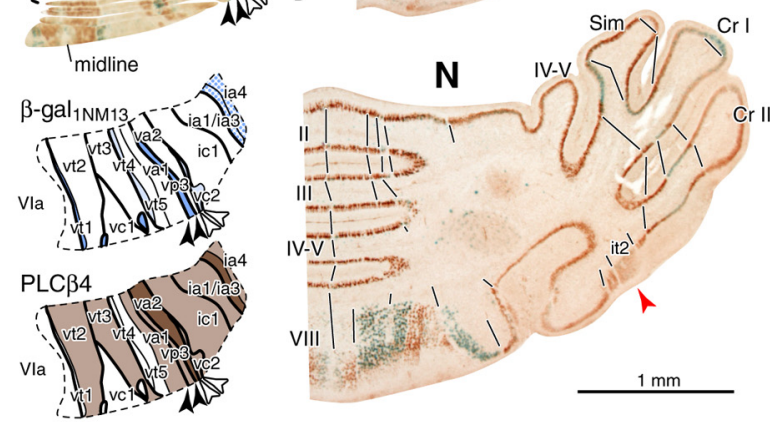

Figure 6. Compartmentalization into stripes in the $\mathrm{P} 6 \mathrm{PC}$ layer. $A$, Lateral view of whole-mount $\beta$-gal ${ }_{1 \mathrm{NM} 13}$ visualization in the $\mathrm{P} 6$ brain. Lines indicate the level and direction of sections shown in $\boldsymbol{B}-\boldsymbol{I}, \boldsymbol{M}, \boldsymbol{N} . \boldsymbol{B}-\boldsymbol{E}$, Coronal sections double-labeled for $\beta$-gal ${ }_{1 \mathrm{NM} 13}$ (blue) and calbindin (brown) $(\boldsymbol{B}, \boldsymbol{C})$ and for $\beta$-gal ${ }_{1 \mathrm{NM} 13}$ (blue) and PLC $\beta 4$ (brown) $(\boldsymbol{D}, \boldsymbol{E})$ at nearly the same level in different mice. Magnified photos in $\boldsymbol{C}$ and $\boldsymbol{E}$ show the same $\beta$-gal ${ }_{1 \mathrm{NM} 13}$-positive stripes bordered by $\mathrm{GC}$ raphes (filled and open arrowheads). $\boldsymbol{F}, \boldsymbol{G}$, Horizontal sections labeled for EphA4 alone, and EphA4 and $\beta$-gal ${ }_{1 \mathrm{NM} 13}$, respectively in different mice. $\boldsymbol{H}$, Coronal section double-labeled for $\beta$-gal ${ }_{1 \mathrm{NM} 13}$ (green, bright field) and Pcdh10 (magenta, fluorescence). $\boldsymbol{I}$, Horizontal section double-labeled for $\beta$-gal ${ }_{1 \mathrm{NM} 13}$ and PLC $\beta 4$.J $\boldsymbol{L}$, Serial section alignment analysis for striped compartments in the PC layer. The PC layer in the rostral wall of lobule Vla was clipped from 25 consecutive sections including that in $I$ and aligned in order $(J)$. Striped compartments (indicated by temporary names), which were recognized by distinct molecular expression profiles and by separation by $\mathrm{GC}$ raphes, were mapped with expression profiles of $\beta$-gal ${ }_{1 \mathrm{NM} 13}(\boldsymbol{K})$ and PLC $\beta 4(\boldsymbol{L})$. GC raphes are indicated by thick lines. $\boldsymbol{M}, \boldsymbol{N}$, Horizontal sections in other levels showing striped compartmentalization of the PC layer distinguished by the $\beta$-gal ${ }_{1 N M 13}$ and PLC $\beta 4$ expression profiles. Straight lines in I, M, N indicate the continuity of PC gaps. Filled and open arrowheads in $I-M$ indicate the same $G C$ raphes in lobule Vla as in $\boldsymbol{C}, \boldsymbol{E}$. Red arrowheads in $\boldsymbol{F}-\boldsymbol{H}, \boldsymbol{M}, \boldsymbol{N}$ indicate the same compartment (it2). Scale bar in $\boldsymbol{H}$ applies to $\boldsymbol{B}, \boldsymbol{D}, \boldsymbol{F}-\boldsymbol{H}$; scale bar in $\boldsymbol{E}$ applies to $\boldsymbol{C}, \boldsymbol{E} ; \boldsymbol{s c a l e}$ bar in $\boldsymbol{N}$ applies to $\boldsymbol{I}, \boldsymbol{M}, \boldsymbol{N}$.

(1NM13) and wild-type embryos and pups were used in these experiments. X-gal labeling of $\beta$-gal ${ }_{1 \mathrm{NM} 13}$ was done in heterozygous embryos and pups. Since we did not observe any differences in shapes or locations of the PC clusters or in any other brain morphology between heterozygous and wild-type embryos and pups, we did not distinguish them unless $\mathrm{X}$-gal labeling was needed.

Concerning these molecules, PLC $\beta 4$ is expressed in aldolase $\mathrm{C}$-negative $\mathrm{PC}$ subsets in the adult cerebellum, but its heterogeneous expression in PC subsets emerges in the embryonic stage (Wilson et al., 2011). Since EphA4 is heterogeneously expressed in certain subsets of inferior olive neurons and their axons (Hashimoto et al., 2012), axonal expression of EphA4 is observed in some PC clusters, in some areas in the cerebellar nuclei, and in some deep medullary areas of the cerebellum. Pcdh10 is expressed heterogeneously in certain PC subsets in the embryonic and early postnatal stages (Hirano et al., 1999). In the present study, we double-labeled each of these markers (magenta or red) with FoxP2 (green) in coronal and horizontal serial sections (Figs. 3, 4). Since these marker molecules are expressed in the soma, processes and/or axons of neurons, labeling of the nucleus of PCs with FoxP2 was remarkably useful for locating the distribution of PCs.

The identification of individual clusters through the combination of molecular expression profiles is shown for sample coronal sections at the similar rostrocaudal level (Fig. $3 A, D, G, J$ ). We identified $\sim 16$ clusters at this level based on the difference in expression profiles of the molecules as mapped in the accompanying drawings (Fig. $3 B, E, H, K$ ). For example, cluster va2 in the center of the section had the highest expression of FoxP2 and PLC $\beta 4$ and a low expression of Pcdh10 and EphA4 (Fig. $3 A, D, G, J$ ). The neighboring clusters icl and ic2 had an expression profile that was different from that of va2. Clusters icl and ic2 could be distinguished from each other since the expression level of Pcdh10 in cluster ic 2 was higher than that in icl and since there was a PC gap between them. Measurements of the expression intensities of molecules in nine clusters in the dorsal aspect (Fig. 3C, F, I,L) indicated that each cluster had distinct expression profiles of these molecules. We identified PC clusters in a similar way in complete serial coronal and horizontal sections of the E17.5 cerebellum.

\section{PC cluster distribution in the E17.5 PC layer}

The arrangement of clusters in the PC layer was different in cerebellar sections at different levels (Fig. 1). This was because each PC cluster had a different shape and because the lateral cerebellum protrudes rostrally. Therefore, to clarify the spatial organization of PC clusters in the whole E17.5 cerebellum, we reconstructed all of the identified clusters in 3D space. We depicted 

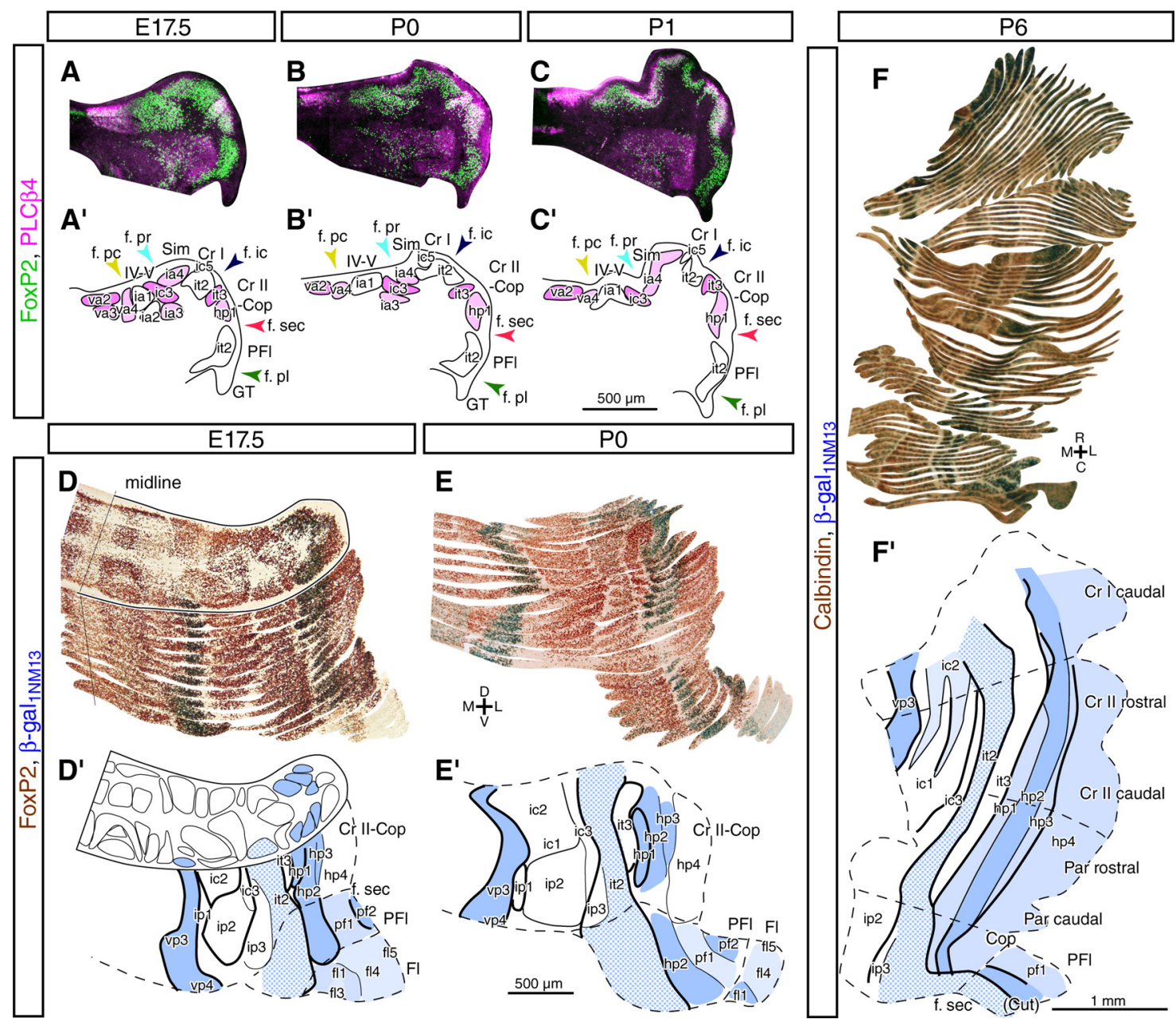

Figure 7. Local rearrangement of $\mathrm{PC}$ subsets from E17.5 to P6.A-C', Coronal sections of the cerebellum at the central level immunostained for FoxP2 and PLC $\beta 4$ at E17.5 (A), P0 (B), and P1 (C). Individual clusters (indicated by temporary names) were mapped in $\boldsymbol{A}^{\prime}-\boldsymbol{C}^{\prime}$. D $-\boldsymbol{F}^{\prime}$, Serial section alignment analysis performed in serial horizontal sections of the posterior hemisphere of the E17.5 $(\boldsymbol{D}), \mathrm{PO}(\boldsymbol{E})$, and P6 $(\boldsymbol{F})$ cerebella, which were double-labeled for $\beta$-gal ${ }_{1 \mathrm{NM} 13}$ and FoxP2 $(\boldsymbol{D}, \boldsymbol{E})$ or for $\beta$-gal ${ }_{1 \mathrm{NM} 13}$ and calbindin-D28k $(\boldsymbol{F})$. Compartmental organization recognized in the analysis is mapped on the schematic drawings $\left(\boldsymbol{D}^{\prime}-\boldsymbol{F}^{\prime}\right)$ along with the expression profiles of $\beta$-gal ${ }_{1 \mathrm{NM13}} \mathrm{GC}$ raphes are indicated by thick lines. The density of PCs was low at the bottom of the secondary fissure in $\boldsymbol{E}(\mathrm{f} . \mathrm{sec})$.

the contours of identified clusters on every serial coronal and horizontal section of the cerebella, in which FoxP2 and $\beta$-gal ${ }_{1 \mathrm{NM} 13}$ were labeled (Fig. $5 A-C$ ). We then imported the drawings to 3D graphics software to reconstruct clusters (Fig. 5D,E). Immature major cerebellar fissures, which were identified by carefully tracing in serial sections, were also added to the scheme. In the resultant 3D reconstruction (Fig. 5F-J; Model 1), we recognized a total of 54 PC clusters. They were located at specific places and had specific shapes and different molecular expression patterns.

Although the 3D reconstruction was originally based on a single E17.5 cerebellum, we carefully compared the cluster arrangement in the $3 \mathrm{D}$ reconstruction with the cluster arrangements in serial coronal and horizontal sections of the cerebella of other E17.5 mice $(n=13)$, which were labeled for various markers, while finalizing the reconstruction. Thus, the cluster arrangement shown in the final reconstruction (Fig. 5; Model 1) was consistent in virtually all E17.5 cases. We did not see any clear interindividual inconsistency in the cluster arrangement, i.e., in general shape or location of the clusters.

While most of the clusters were elongated in the longitudinal (rostrocaudal) direction across immature fissures, some clusters were localized within a single immature lobule. Particularly, vermal lobule X (Fig. 5I), flocculus (Fig. 5I) and lateral crus I (Fig.
$5 H$ ) tended to have clusters that did not extend beyond immature fissures. Some clusters were positioned on top of other clusters. This complex positioning indicated the rearrangement of clusters during development after E17.5 (later section).

To identify these clusters in the present study, they were classified according to the longitudinal (vermis, v; pars intermedia, i; hemisphere, h; used as the first character) and transverse (anterior, a; central, c; posterior, $\mathrm{p}$; throughout a-p, t; used as the second character) cerebellar subdivisions within which they were mainly located. Clusters that were mainly located in the paraflocculus (pf), flocculus (fl) and nodulus (no) were classified separately. Clusters in each class were then numbered from medial to lateral (the third character). For example, cluster val was the most medial cluster in the vermal anterior area.

\section{Compartmentalization of the P6 PC layer according granule cell raphes and molecular expression patterns}

To follow the clustered compartmental organization of the E17.5 PC layer to later postnatal stages, we now focused on the P6 cerebellum, in which the basic adult-type morphology began to be established. In the P6 cerebellum, (1) the PC layer became single-cell thick, (2) foliation of major lobules (vermal lobules I, 
A

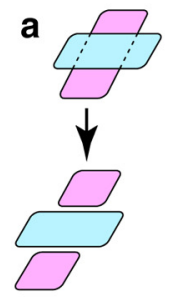

b
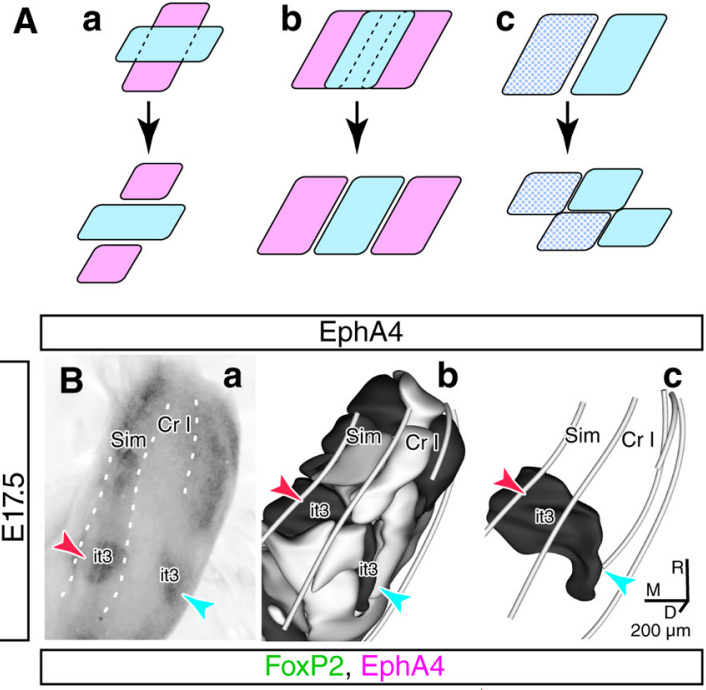

足
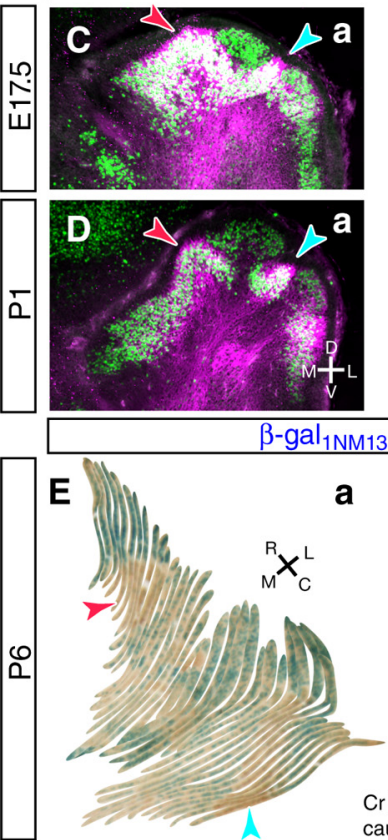
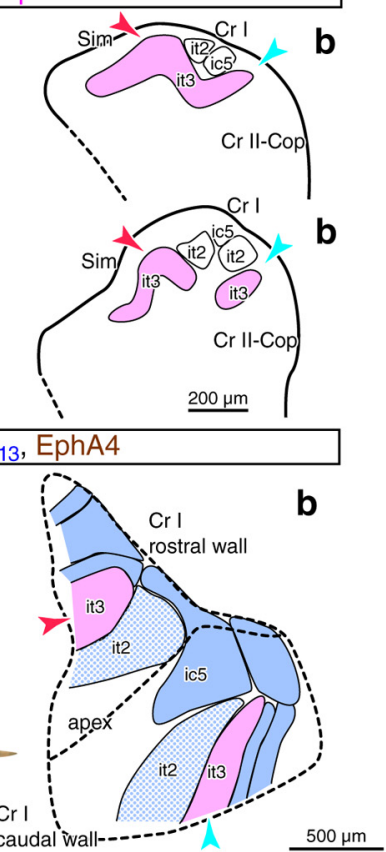
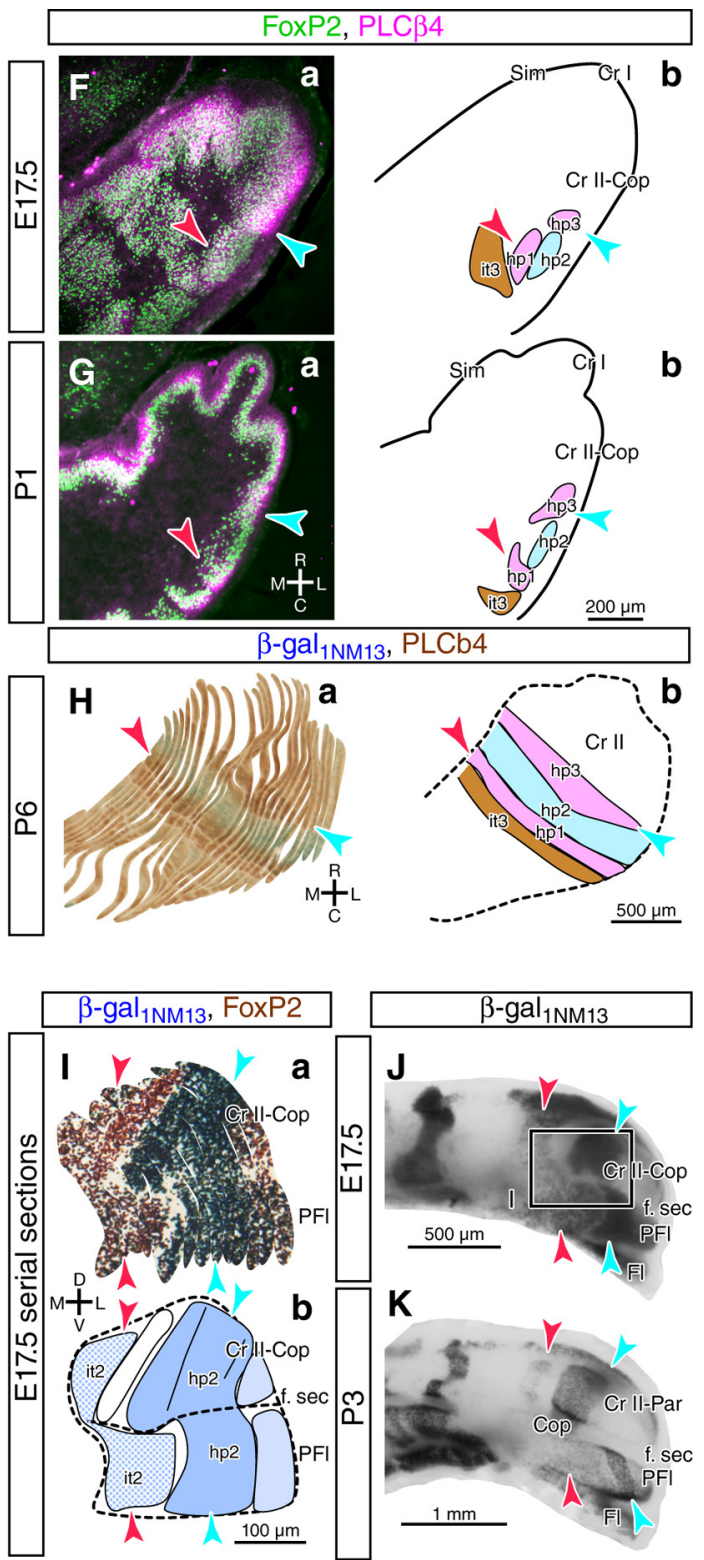

Figure 8. Modes of transformation of PC subsets during peri- and postnatal cerebellar development. $\boldsymbol{A}$, Schematics of modes of transformation; longitudinal split ( $\boldsymbol{a}$ ), transverse slide ( $\boldsymbol{b}$ ), and transverse twist (c). $\boldsymbol{B}-\boldsymbol{E}$, "Longitudinal split"-type transformation in EphA4-positive cluster it3 in crus I. Cluster it3 in the E17.5 cerebellum was shown in dorsal view of whole-mount EphA4 immunostaining $(\mathbf{B a})$ and in three-dimensional reconstruction with $(\boldsymbol{B} \boldsymbol{b})$ and without $(\boldsymbol{B C})$ other clusters. Coronal sections immunostained for FoxP2 and EphA4 at E17.5 (C) and P1 (D) shows split of cluster it3 at P1. Serial section alignment analysis for horizontal sections labeled for $\beta$-gal ${ }_{1 \mathrm{NM} 13}$ and EphA4 at P6 (E) shows separation of it3. Cluster/compartment it3 is colored consistently in mappings $(\boldsymbol{C} \boldsymbol{b}, \boldsymbol{D} \boldsymbol{b}, \boldsymbol{E} \boldsymbol{b})$. Red and cyan arrowheads indicate the rostral and caudal parts of cluster/compartment it3, respectively. $\boldsymbol{F}$ - $\boldsymbol{H}$, "Transverse slide"-type transformation in four clusters/ compartments in crus II (it3, hp1, hp2, and hp3). Horizontal sections of the central cerebellum (lobules IV-V, crus I, and crus II-paramedian lobule-copula pyramidis) immunostained for FoxP2 and PLC $\beta 4$ at E17.5 $(\boldsymbol{F a})$ and P1 $(\mathbf{G a})$ shows change of cluster disposition as depicted in the accompanying drawings $(\boldsymbol{F b}, \mathbf{G b})$. Serial section alignment analysis for horizontal sections labeled for $\beta$-gal ${ }_{1 N M 13}$ and PLC $\beta 4$ at P6 $(\boldsymbol{H})$ shows striped alignment of the four compartments in crus II. These compartments are colored consistently in mappings $(\boldsymbol{F} \boldsymbol{b}, \boldsymbol{G} \boldsymbol{b}, \boldsymbol{H b})$. Red and cyan arrowheads indicate compartments hp1 and hp3, respectively. $\boldsymbol{I - K}$, "Transverse twist"-type transformation in the paraflocculus. Serial section alignment analysis shows two compartments (it2 and hp2) were continuous across the secondary fissure at E17.5 (Ia) as depicted in the accompanying drawing (Ib). Since these subsets consistently express $\beta$-gal ${ }_{1 \mathrm{NM} 13 \text {, }}$, they were followed in the caudal view of whole-mount $\beta$-gal ${ }_{1 \mathrm{NM} 13}$ visualization at E17.5 $(\boldsymbol{J})$ and $\mathrm{P} 3(\boldsymbol{K})$. The ventral part in the paraflocculus was twisted rostrolaterally in these clusters. Red and cyan arrowheads indicate clusters/ compartments it2 and hp2, respectively.

II, III, IV-V, VI, VII, VIII, IX and X, and hemispheric lobules IV-V, simple lobule, crus I, crus II, paramedian lobule and copula pyramidis) became recognizable, and (3) the $\beta$-gal ${ }_{1 \mathrm{NM} 13}$ expression pattern was fully developed (Fig. $1 B$; Furutama et al., 2010). Most embryonic marker molecules of PC subsets lose a clear compartmental expression pattern before P6, and most adult marker molecules of PC subsets are not yet clearly expressed at P6 (see Introduction). However, $\beta$-gal ${ }_{1 \mathrm{NM} 13}$ (Fig. $1 D$; Furutama et al., 2010), PLC $\beta 4$ (Marzban et al., 2007), and Pcdh10
(Hirano et al., 1999) are clearly expressed in a specific PC subset at $\sim$ P6. In addition, granule cell (GC) raphes, which are narrow PC-free spaces filled with GCs in the PC layer, have been observed to subdivide the PC layer into multiple divisions at P6 (Luckner et al., 2001). GC raphes can be visualized by labeling all PCs with calbindin-D28k (Karam et al., 2001), which is expressed in all PCs at P6. We carefully examined the positional relationships of expression patterns of these markers by double-labeling $\beta$-gal ${ }_{1 \mathrm{NM} 13}$ and PLC $\beta 4$, calbindin-D28k, EphA4 or Pcdh10 in coronal and 


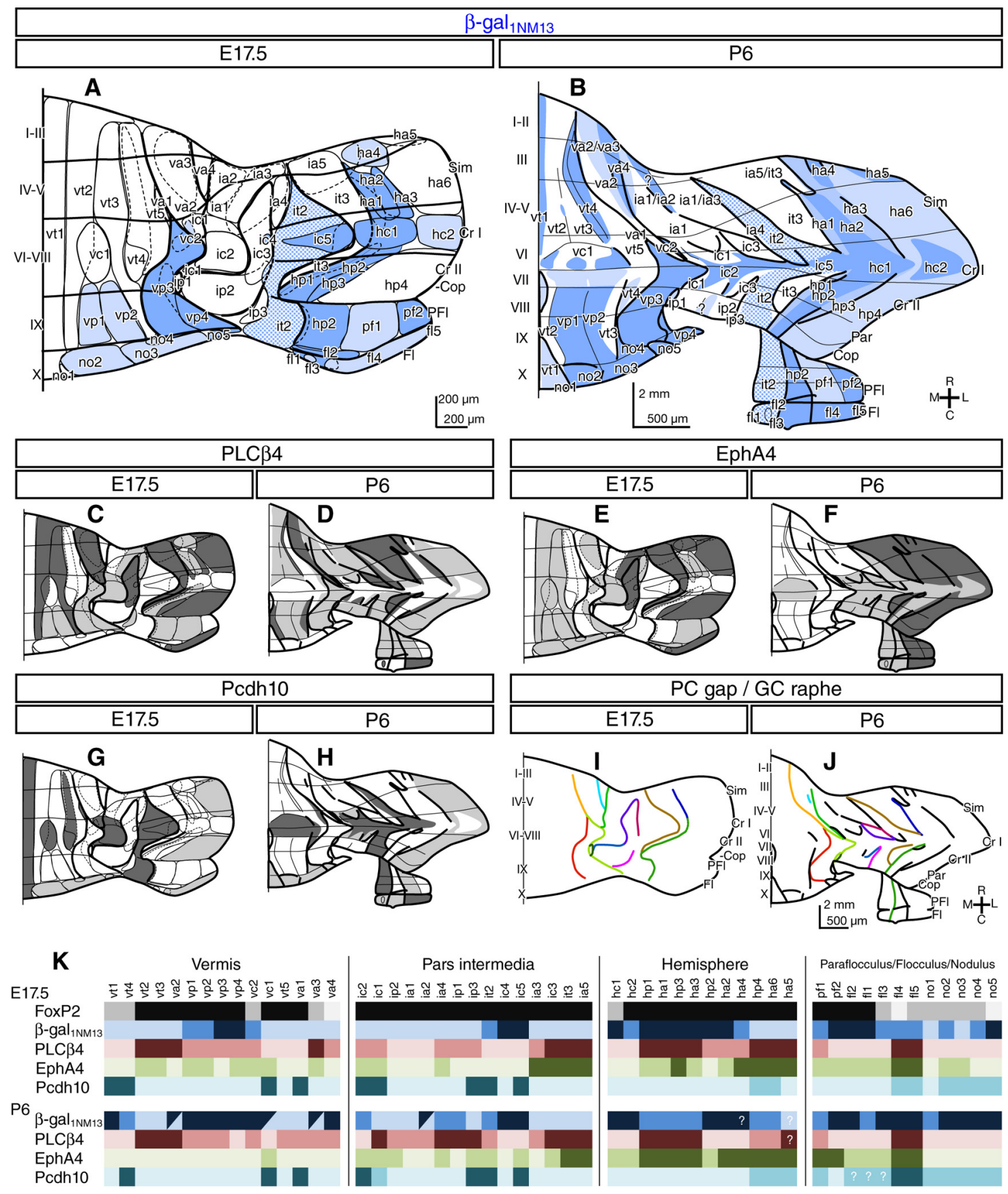

Figure 9. Correspondence of molecular expression patterns in compartments throughout the entire cerebellar cortex between E17.5 and P6. A-H, Expression patterns of $\beta$-gal ${ }_{1 \mathrm{NM} 13}$, PLC $\beta 4$, EphA4 and Pcdh10 in PC clusters/compartments mapped on the unfolded scheme of the cerebellar cortex at E17.5 and P6. The labeling intensity of molecules is indicated by the darkness of colors. Compartment boundaries in the deep PC layer are shown with dashed curves in the E17.5 schemes. Thick lines indicate PC gaps and GC raphes. I, J, Clear PC gaps at E17.5 and GC raphes at P6. Each color indicates correspondence. $\boldsymbol{K}$, Intensity of the molecular expression in individual PC subsets represented by light, medium, or dark colors in the E17.5 (top) and P6 (bottom) cerebellum. Darker colors indicate higher expression intensity. Slashed cells in $\mathrm{P} 6 \beta$-gal ${ }_{1 \mathrm{NM} 13}$ expression mean that the subset may be separated into multiple small subsets that have different $\beta$-gal ${ }_{1 \mathrm{NM} 13}$ expression profiles. Cells with "?" in P6 $\beta$-gal ${ }_{1 \mathrm{NM} 13}$ expression means that the observation was not clear.

horizontal serial sections (Fig. 6) to clarify the compartmentalization of the P6 PC layer.

Expression patterns of these markers were closely related to GC raphes in the P6 PC layer. For example, two $\beta$-gal ${ }_{1 \mathrm{NM} 13^{-}}$ positive stripes (vp3 and vc2) were consistently observed in the rostral wall of the simple lobule in the pars intermedia. These stripes were bordered by GC raphes (Fig. $6 C$, filled and open arrowheads). The area between these stripes showed the intense expression of PLC $\beta 4$ (Fig. 6E). Stripes defined by EphA4 and Pcdh10 expression profiles were also bordered by GC raphes (Fig.
$6 F-H)$. Each stripe had a specific profile of molecular expression. For example, the relatively wide stripe in crus II and the paramedian lobule indicated by the red arrowheads (it2) in Figure $6 \mathrm{~F}-\mathrm{H}$, $M$, and $N$ was $\beta$-gal ${ }_{1 \mathrm{NM} 13}$-weakly positive, PLC $\beta 4$-weakly positive, EphA4-weakly positive and Pcdh10-positive. Spatial patterns of molecular expression and GC raphes were continuous in the longitudinal direction across lobules (Fig. 6I, M,N, lines), but were also clearly different in separate lobules. However, since the cerebellar cortex is deeply foliated at P6, it was difficult to examine the continuity of these patterns in separate sections. There- 
fore, to further clarify the spatial patterns of molecular expression and GC raphes within a single lobule and across lobules, we developed a method which we call "serial section alignment analysis (SSAA).” The PC layer in a particular area (the rostral wall of lobule VIa in the vermis and pars intermedia, in the case of Fig. $6 J$ ) was clipped off in every serial section. The clipped PC layers were then aligned in order (Fig. $6 \mathrm{~J}$ ) to visualize the longitudinal continuity of molecular expression patterns and GC raphes. Mapping of these patterns in SSAA revealed that the rostral wall of lobule VIa in the vermis and pars intermedia was divided into 14 stripes that were distinguished by PLC $\beta 4$ and $\beta$-gal ${ }_{1 \mathrm{NM} 13}$ expression patterns and were often separated by GC raphes (Fig. $6 K, L)$. The particular stripes and GC raphes indicated in Figure 6, $C$ and $E$, were clearly demonstrated in SSAA (Fig. $6 \mathrm{~J}, L$, filled and open arrows). We performed SSAA in all lobules in the P6 cerebellum to map stripes distinguished by molecular expression patterns and GC raphes in the entire cerebellar cortex (see Consistency of compartmentalization of the PC layer between E17.5 and P6, below).

\section{Individual E17.5 PC clusters became distinct P6 stripes}

The organizations of the E17.5 and P6 PC layers were clearly different. The E17.5 PC layer was composed of PC clusters, some of which were located beneath others (earlier section). Therefore, we took a close look at how the E17.5 PC layer was transformed to the P6 PC layer, which has a striped compartmentalization. We first compared the compartments in the PC layer, which were labeled for FoxP2 and PLC $\beta 4$, in the coronal sections of the central level of the cerebellum at three successive days from E17.5 to P1 (Fig. 7A-C). In this level of the coronal section, PLC $\beta 4$ positive (va2 and va3), -lightly positive (va4), -negative (ial and ia2), -positive (ic3), -lightly positive (ia3 and ia4), -negative (ic5 and it2), -positive (it3), and -lightly positive (hp1) clusters could be identified from medial to lateral at E17.5. Nearly the same set of clusters was seen at $\mathrm{P} 0$, and $\mathrm{P} 1$, although a few clusters such as ia2 and ia3 gradually disappeared in a single section. At E17.5, clusters were generally thick in the radial direction and sometimes located beneath other clusters. At P0 and then at P1, clusters became generally thinner and less frequently located beneath other clusters, which was caused by the tangential rearrangement of PC clusters. Thus, the PC layer became more flattened and clusters became more aligned on a single plane in the PC layer at $\mathrm{P} 1$ than at E17.5. This process represented the main developmental transformation of the PC layer during this period. Furthermore, this process of the transformation of the PC layer was accompanied by longitudinal expansion of the PC layer or the cerebellar cortex and foliation of the cerebellar cortex. Indeed, the five fissures of the cerebellar cortex, which were recognized as tiny dents at E17.5, became gradually prominent at P0 and P1 (Fig. $7 A^{\prime}-C^{\prime}$, colored arrowheads).

Next, we examined rearrangement of the PC layer compartmentalization from E17.5 to P6. SSAA was applied in the posterior hemisphere (crus II and the paramedian lobule) at E17.5, P0, and P6 (Fig. 7D-F). At E17.5, crus II and the paramedian lobule formed a single bulge, where two intensely $\beta$-gal INM13 $_{\text {-positive }}$ clusters were seen (vp3-vp4 and hp1-hp2-hp3). Between these clusters, three sets of clusters separated by PC gaps were arranged

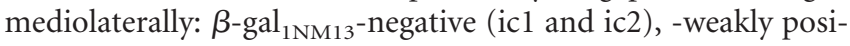
tive (it2), and -negative (it3) clusters (Fig. $7 D, D^{\prime}$ ). SSAA at P0 showed nearly the same organization of clusters in the posterior hemisphere (Fig. $7 E, E^{\prime}$ ). At P6, SSAA in the posterior hemisphere became more complicated since crus II, the paramedian lobule and copula pyramidis made distinct folia. However, the two intensely $\beta$-gal ${ }_{1 \mathrm{NM} 13}$-positive stripes (vp3-vp4 and hp1-hp2-hp3) were clearly seen. Between these stripes, three sets of stripes separated by GC raphes were arranged mediolaterally: a $\beta$-gal ${ }_{1 \mathrm{NM} 13}$-negative (ic1 and ic3), -weakly positive (it2), and -negative (it3) stripes (Fig. $\left.7 F, F^{\prime}\right)$. These results indicated that the compartmental organization of the PC layer in the posterior hemisphere was almost the same between E17.5 and P6. Distinct clusters at E17.5 were transformed to distinct stripes at P6. Furthermore, all of the PC gaps at E17.5 were transformed to GC raphes at P6. Therefore, we used the same nomenclature to designate PC stripes at P6 as that for the clusters at E17.5. Similar analyses in other areas basically gave consistent results.

\section{Rearrangement of PC clusters}

Peri- and postnatal growth of the cerebellar cortex is accompanied by the general rostrocaudal dispersal of PCs (Sgaier et al., 2005). In addition to this rostrocaudal dispersal, our analysis showed that development of the PC layer also involved the distinct tangential rearrangement of PC subsets (Miyata et al., 2010). Closer observation revealed three particular types of rearrangement of PC subsets, from a clustered and layered configuration to a striped and flattened configuration (Fig. $8 \mathrm{~A}$ ).

Some clusters that were located beneath other clusters were separated into the two portions that migrated rostrally and caudally ("longitudinal split," Fig. 8Aa). The example in Figure $8 B-E$ focused on an EphA4-positive cluster (it3) in the central hemisphere (crus I). Whole-mount immunostaining for EphA4 labeled not the whole cluster it3, but rather its two separate portions that were close to the cerebellar surface at E17.5 (Fig. 8Ba, red and cyan arrowheads). However, 3D analyses from serial sections showed that these portions were parts of the same cluster (it3) that was long in the mediolateral direction and located, at its middle, beneath an EphA4-negative cluster (it2 and ic5) at E17.5 (Fig. $8 B b, c, C$ ). This cluster (it3) was split mediolaterally at P1 beneath other clusters (it2 and ic5) at P0 (Fig. $8 D$ ). Since the lateral part of the cerebellum is curved to protrude rostrally in this developmental stage, the mediolateral direction in the coronal section partly represented the longitudinal (rostrocaudal) direction. At P6, these clusters became separate EphA4-positive stripes in the rostral and caudal wall of crus I (Fig. $8 E$, red and cyan arrowheads).

Some clusters that were located beneath other clusters migrated medially or laterally to completely face the surface of the PC layer ("transverse slide," Fig. $8 A b$ ). The example in Figure $8 \mathrm{~F}-\mathrm{H}$ shows the transformation of four clusters (it3, hp1, hp2, and hp3) in the posterior hemisphere (crus II), among which cluster hp2 was PLC $\beta 4$-negative and clusters it 3 and hp1 were separated by a clear PC gap (Fig. $8 F$, red and cyan arrowheads). Clusters hp1 and hp3 were mostly and partially located beneath cluster hp2, respectively, at E17.5. They gradually migrated medially and laterally, respectively, at P1 (Fig. 8G). They were completely located on the medial and lateral sides of stripe hp2 (former cluster hp2) at P6 (Fig. $8 \mathrm{H}$, red and cyan arrowheads). The expression pattern of PLC $\beta 4$ was consistent in these clusters from E17.5 to P6.

Clusters located across the secondary fissure (boundary of the caudal hemisphere and paraflocculus) became nearly separated since the paraflocculus was rotated rostrally in the early postnatal stage ("transverse twist," Fig. 8 Ac). At E17.5, some $\beta$-gal ${ }_{1 \mathrm{NM} 13^{-}}$ positive clusters were continuous across the bottom of the secondary fissure (Fig. 8I, it2 and hp2; red and cyan arrowheads). The parafloccular parts of these clusters were shifted laterally and rotated rostrally while the rest of these clusters remained at their 
Table 2. Comparison of the embryonic cerebellar compartments with previous reports by marker and age

\begin{tabular}{|c|c|c|c|c|c|c|c|c|c|c|c|c|c|c|c|c|c|c|c|c|c|c|c|c|c|c|c|c|c|c|c|c|}
\hline Reference $^{a}$ & Marker & Age & Verr & & & & & & & & & & & & & & & Pars i & termed & & & & & & & & & & & & & \\
\hline 1 & $\begin{array}{l}\beta \text {-gal } \\
\quad \text { Pcdh10 } \\
\text { Pcdh }\end{array}$ & E17.5 & vt1 & vt 4 & vt2 & vt3 & va2 & vp1 & vp2 & vp3 & vp4 & vc2 & vc1 & vt5 & va1 & va3 & va4 & ic2 & ic1 & ip2 & ia1 & ia2 & ia4 & ip1 & ip3 & it2 & ic4 & ic5 & ia3 & ic3 & it3 & ia5 \\
\hline 2 & Birthdate & E18.5 & 1 & - & 2 & $2 ?$ & 4 & 2 & 2 & 4 & - & - & 2 & 3 & - & - & - & 5 & - & - & 5 & 5 & $6 ?$ & - & - & 6 & 5 & $6 ?$ & 5 & 5 & 7 & 7 \\
\hline 3 & En1 & E17.5 & B1 & - & B1 & B1 & - & - & - & - & - & - & - & B2 & - & - & - & - & - & - & - & - & - & - & - & B3 & - & B3 & - & - & - & - \\
\hline 4 & En2 & E17.5 & B1 & - & B2 & B2 & - & - & - & - & - & - & - & - & - & - & B3 & - & - & - & B3 & B3 & - & - & - & B4 & - & B4? & B3 & B3 & - & - \\
\hline 5 & Pcp2-CreER-IRES-hAP & E18.5 & - & - & 1 & - & - & 1 & $1 ?$ & - & - & - & 1 & 2 & - & - & - & 3 & $3 ?$ & - & - & - & - & - & - & $4-5$ & - & 4 & - & $3 / 4$ & - & - \\
\hline 6 & Pcp2-lacz ${ }^{b}$ & E17 & - & - & 1 & $2 ?$ & - & 1 & $2 ?$ & 3 & - & - & $1 / 2$ & - & - & - & - & $4-5$ & $4-5 ?$ & - & - & - & - & - & - & - & - & - & - & - & - & - \\
\hline 7 & Neurogranin & E17 & - & - & 1 & 2 & - & - & - & - & - & - & - & 3 & - & - & - & - & - & - & - & - & - & - & - & - & - & - & - & $4 ?$ & - & - \\
\hline 8 & PKG & $\mathrm{E} 19$ (rat) ${ }^{d}$ & 1 & - & I & I & - & - & - & - & - & - & - & - & $\|$ & - & - & III & III & - & - & - & - & - & - & IV & - & - & - & - & - & - \\
\hline 9 & Tritium-labeling $^{e}$ & E21 (rat) & - & C1? & - & $(4 ?$ & - & - & C2? & - & - & - & - & C5? & - & - & - & - & - & - & - & - & - & - & - & - & - & - & - & - & - & - \\
\hline
\end{tabular}

${ }^{a}$ Reference key is as follows: 1, The present study; 2, Hashimoto and Mikoshiba (2003); 3, 4, Wilson et al. (2011); 5, Sillitoe et al. (2009); 6, Fig. 2 of Oberdick et al. (1993); 7, Larouche et al. (2006); 8, Fig. 4B of Wassef et al. (1984); 9, Fig. 12-10 of Altman and Bayer (1997). Since the previous studies were based on a small number of sections and/or whole-mount preparations, these studies seems to be inadequate for identifying the complete set of compartments that were identified in the present study. Correspondence was inferred based on the shape and localization of the clusters and the reported expression patterns of marker molecules. In some cases, when the nomenclature was not consistent through the study, we focused on a particular figure of the study.

${ }^{b} 0.5 \mathrm{~kb}$ promoter line, L7BG3-L12.

'PKG is CGK (cyclic 3', 5' -guanosine monophosphate-dependent protein kinase).

${ }^{d}$ Studies were performed in the mouse unless "rat" is added.

${ }^{e}$ The section was counterstained with cresyl violet and toluidine blue.

original positions (Fig. $8 \mathrm{~J}, \mathrm{~K}$ ). The $\beta$-gal INM13 $_{3}$ expression pattern of these clusters was consistent during this period.

\section{Consistency of compartmentalization of the PC layer between E17.5 and P6}

By applying SSAA in various areas at E17.5, P0, P1, P3, and P6, and by considering the rearrangement of clusters, we followed almost all of the individual clusters in the E17.5 PC layer to individual stripes in the P6 PC layer throughout the entire cerebellar cortex. All of the clusters and stripes as well as PC gaps (GC raphes) are depicted in the schemes of the unfolded cerebellar cortex at E17.5 and P6 (Fig. 9A,B). Clusters and stripes were colored according to the expression patterns of $\beta$-gal ${ }_{1 \mathrm{NM} 13}$ and other markers. The scheme for the E17.5 PC layer (Fig. 9A), which shows the same organization of clusters as in the $3 \mathrm{D}$ scheme in Figure 5, was more complicated than the scheme for the P6 PC layer to show the overlapping of multiple clusters. At $\mathrm{P} 6$, the PC layer is mono-cell thick and divided by GC raphes. GC raphes were almost always located on the boundaries between stripes that expressed molecules differently at P6 and could be traced back to PC gaps at E17.5 (Fig. 9I,J).

Individual stripes at P6 were located approximately in the same position as their original PC clusters at E17.5 in relation to neighboring stripes and fissures. The molecular expression patterns in clusters or stripes were not exactly the same between E17.5 and P6, since several more stripes expressed $\beta$-gal ${ }_{1 \mathrm{NM} 13}$ at P6 (Fig. 9A, $B$ ) and the expression levels of molecular markers in individual stripes sometimes changed between E17.5 and P6. However, the consistently high expression of $\beta$-gal INM13 $_{3}$, PLC $\beta 4$, EphA4 or Pcdh10 in the compartments vp3, vp4, ic4, ic5, hc1, ha2, hp2 pf2 and no5; vt2, vt3, va2, ic3, hp1, ha1, hp3, ha3, fl4 and fl5; it3, ia5, hp3, ha4, hp4, ha6, ha5, fl4 and fl5; vt4, vc1, va1, ic2, ip3, it2, ic5 and it2, respectively, was particularly useful for following these compartments (Fig. 9A-H). The consistently moderate or absent expression of these markers was also useful for following compartments. However, we could not follow a small number of clusters (va3, ia1, ia2, ia3, and ia5) since they could not be clearly distinguished from neighboring clusters during development from E17.5 to P6. This was because the PC gaps that separated them gradually became ambiguous without developing to GC raphes and because the difference in the expression profiles of molecular markers was not very clear between them. They may represent a group of PCs migrating to join a neighboring cluster.
Finally, we summarized the expression patterns of FoxP2, $\beta$-gal ${ }_{1 \mathrm{NM} 13}, \mathrm{PLC} \beta 4, \mathrm{EphA} 4$, and Pcdh10, in all clusters at E17.5 and all stripes at P6 (Fig. 9K). Although the molecular expression profile of each cluster was not always consistent during development, the original clusters were identified for almost all of the stripes at P6. Several sets of clusters showed similar molecular expression profiles, which were arbitrarily positioned close in the diagram. However, many clusters had unique molecular expression profiles. Although the molecular expression profiles did not seem to be random, it was generally difficult to find a simple relationship between molecular expression profiles and spatial arrangements of PC clusters. However, we could observe that stripes located in the rostral and caudal parts of the cerebellum at a similar mediolateral position sometimes showed similar molecular expression patterns, such as ha1-hp1, ha2-hp2, and ha3-hp3, which resembles the feature of aldolase $\mathrm{C}$ expression in adult stripes (Sugihara and Shinoda, 2007).

\section{Discussion}

The present study has shown that the embryonic PC layer already has fine compartmentalization into $\sim 50$ clusters of PCs at E17.5. Individual E17.5 clusters generally develop into individual postnatal striped compartments through a set of rearrangement processes. The modes of developmental rearrangement of E17.5 PC clusters to striped P6 compartments have also been revealed. Thus, the present study has critically related the E17.5 clustered organization to the adult-type striped organization in the developing cerebellar cortex. It still remains unclear how and when the clustered PC compartments are formed before E17.5. The functional implication of clustered compartmentalization and its rearrangement processes will be discussed.

\section{PC clusters in the E17.5 cerebellar cortex}

PCs are generated during the period from E10.5 to E12.5 in the neuroepithelium of the cerebellar primordium (Hashimoto and Mikoshiba, 2003). Early PCs migrate radially (Rakic and Sidman, 1970; Altman and Bayer, 1985) and/or tangentially from caudal to rostral (Miyata et al., 2010) toward areas beneath the outer surface of the cerebellar primordium. In the late embryonic period $\sim$ E17.5 (E19 in the rat), PCs are densely distributed in the area beneath the outer surface (80-300 $\mu \mathrm{m}$ deep) of the entire cerebellar primordium (Wassef and Sotelo, 1984), and form the 
Table 2. (Continued)

\begin{tabular}{|c|c|c|c|c|c|c|c|c|c|c|c|c|c|c|c|c|c|c|c|c|c|c|c|}
\hline \multicolumn{12}{|c|}{ Hemisphere } & \multicolumn{2}{|c|}{ Paraflocculus } & \multicolumn{5}{|c|}{ Flocculus } & \multicolumn{5}{|c|}{ Nodulus } \\
\hline hc1 & hc2 & hp1 & ha1 & hp3 & ha3 & hp2 & ha2 & ha4 & hp4 & ha6 & ha5 & $\mathrm{pf1}$ & pf2 & $\mathrm{fl} 2$ & $\mathrm{fl} 1$ & $\mathrm{fl} 3$ & $\mathrm{fl} 4$ & $\mathrm{fl} 15$ & no1 & no2 & no3 & no4 & no5 \\
\hline- & - & 8 & 8 & 8 & 8 & 8 & 8 & - & 8 & - & - & 8 & - & - & - & - & - & - & - & - & - & - & - \\
\hline- & - & - & - & - & - & - & - & - & - & - & - & - & - & - & - & - & - & - & - & - & - & - & - \\
\hline - & - & - & - & - & - & - & - & - & - & - & - & - & - & - & - & - & - & - & - & - & - & - & - \\
\hline - & - & - & - & - & - & - & - & - & - & - & - & - & - & - & - & - & - & - & - & - & - & - & - \\
\hline- & - & - & - & - & - & $7 ?$ & $7 ?$ & - & - & - & - & - & - & - & - & - & - & - & - & - & - & - & - \\
\hline- & - & - & - & - & - & - & - & - & - & - & - & - & - & - & - & - & - & - & - & - & - & - & - \\
\hline - & - & - & - & - & - & - & - & - & - & - & - & - & - & - & - & - & - & - & - & 1 & - & - & - \\
\hline- & - & - & - & - & - & - & - & - & - & - & - & - & - & - & - & - & - & - & - & C3 & - & - & - \\
\hline
\end{tabular}

immature PC layer. The present study has revealed how PC subsets are organized at this stage.

In the present study, we looked at serial sections of the entire cerebellum in which we had specifically labeled PCs by the immunostaining of FoxP2 in combination with other marker molecules to identify and reconstruct what appear to be all of the E17.5 clusters. The cluster arrangement that we demonstrated was consistently seen in all examined cases. Previous reports on the compartmentalization of the embryonic PC layer (Wassef and Sotelo, 1984; Oberdick et al., 1993; Millen et al., 1995; Altman and Bayer, 1997; Hashimoto and Mikoshiba, 2003; Larouche et al., 2006; Sillitoe et al., 2009; Wilson et al., 2011) were based on a small number of sections and/or whole-mount preparations. Therefore, these studies could not clarify the detailed organization of PC clusters throughout the entire embryonic cerebellum. However, we can infer how the clusters reported in these previous studies correspond to the clusters identified in the present study (Table 2).

\section{Functional significance of clustering of PCs}

Neuronal clustering may be essential in development of compartmentalization for topographic afferent and efferent connections in some brain areas. In the ventral horn of the spinal cord, motor neurons that innervate different muscles emerge as clusters (Jessell et al., 2011), which is essential for correct innervation of afferent axons (Sürmeli et al., 2011). Projections of climbing fibers and mossy fibers, which have compartment-specific topography in adult (Sugihara and Shinoda, 2004, 2007; Quy et al., 2011), begin to form in the cluster stage (Sotelo et al., 1984; Ashwell and Zhang, 1992; Paradies and Eisenman, 1993). It is not clear how precise topography the immature projections of climbing and mossy fibers have at this stage. However, we speculate that $>50$ clusters, which have different expression profiles of molecules, may secure the development of proper topographic connections in the cerebellar cortex through molecular matching between PCs and afferent axons (Sotelo, 2004).

Indeed, EphA4, a molecular marker that was used in the present study, is one of the Eph receptor tyrosine kinases, which play a role in axonal guidance, boundary formation and cell migration in the retinotectal system and other CNSs (Flanagan and Vanderhaeghen, 1998). In our case, EphA4 may be expressed mainly in olivocerebellar axons, which have basically become established by the perinatal period with sufficiently precise topographic patterns. Cadherin family molecules are involved in cell adhesion, formation of axonal projection and synaptogenesis (Redies, 2000). In addition to EphA4 and Pcdh10, expression of which the present study examined, other Eph receptor tyrosine kinases and cadherin family molecules are expressed in a compartment (or cluster-)-specific manner in the immature mouse cerebellar cortex (Karam et al., 2000; Neudert et al., 2008). These molecules are also expressed cluster-specifically in spinal motor neurons and are involved in formation of topographic sensorimotor neuronal circuits (Flanagan and Vanderhaeghen, 1998; Price et al., 2002). Thus, cluster formation is one of the basic mechanisms that enable organized topographic connection of growing axons in the CNS. Factors that are involved in formation of clusters, as demonstrated in the spinal cord (Dasen et al., 2008), may be studied in the cerebellum.

\section{Development of the PC layer after E17.5}

Whereas PC clusters are arranged into multiple layers at E17.5 (designated the "cluster stage" here), all of the clusters are located within a roughly flat $\mathrm{PC}$ layer at $\sim \mathrm{P} 1$ as a result of the rearrangement of PC clusters. The PC layer is still multi-cell thick at this stage (designated the "multicell stage"). With the further rostrocaudal expansion of the cerebellum, the PC layer becomes monocell thick at $\sim$ P6 (Altman and Bayer, 1997). GC raphes, which are remnants of the $\mathrm{PC}$-free space between clusters as revealed in this study, separate the PC layer at this stage (designated the "raphemonolayer stage"). GC raphes disappear later (Luckner et al., 2001) by adulthood (designated "developed stage"). As revealed in the present study, these transformations of the PC layer are closely related to the development of the compartmental organization in the PC layer.

The reorganization of clusters into monolayered stripes is unique to the cerebellar cortex. Although we have shown some details of this reorganization, mechanisms for this process may be studied further. This reorganization process may be accompanied by refinement of axonal projections. Climbing and mossy fiber projections suffer significant pruning and reorganization of the projection in the same period (Arsénio-Nunes and Sotelo, 1985; Sotelo, 2004; Sugihara, 2005). Intensity of molecular expression changes dynamically in clusters during the period between E17.5 and P6. For example, EphA4 expression generally decreases, which might reflect pruning of climbing fibers that express EphA4. A gradient in expression intensity of molecules may be involved in the refinement of axonal projection patterns at the local level, as demonstrated in the tectum (Flanagan and Vanderhaeghen, 1998). A similar mechanism may be involved in refinement of the afferent axonal projection pattern at the substripe or subcompartment level in the cerebellum. Assuming this refinement occurs, each cluster or stripe may be subdivided into multiple subareas that are involved in related but different cerebellar functions.

\section{GC raphes and PC gaps in the developing cerebellar cortex}

GC raphes were first reported in the chick embryonic cerebellum (Feirabend, 1990). They appear between E8 and E15 in the chick cerebellar cortex as PC-poor gaps in the PC layer and correspond to the boundaries between PC subsets with distinct gene expression profiles (Feirabend, 1990; Lin and Cepko, 1998; Karam et al., 
2000). GC raphes also border the PC subsets in the mammalian cerebellar cortex (P0-P6 in mice; Luckner et al., 2001; P0-P9 in rats, Ji and Hawkes, 1995; E80-E90 in monkeys, Karam et al., 2001).

While clarifying the striped compartmentalization of the P6 cerebellum, we have identified GC raphes at particular boundaries between PC stripes of distinct molecular expression profiles. We have also revealed that the origin of GC raphes in mice is the PC-free spaces that separate PC clusters in the E17.5 PC layer. Thus, GC raphes can be regarded as an essential landmark structure in the developmental transformation of the PC layer from the cluster stage to the raphe-monolayer stage. Concerning their functional role, the aggregation of GCs and PCs into PC-free spaces and PC clusters, respectively, is presumably mediated by the adhesive function of cadherin family molecules, which are consistently expressed by GCs and PCs (Luckner et al., 2001). Thus, we suppose that both structures may be involved in the transformation of the PC layer from the cluster stage to the monolayer stage between E17.5 and P6.

\section{Cortical organization reflected in the E17.5 cluster arrangement}

Based on the adult rodent aldolase $\mathrm{C}$ expression pattern and projection patterns of olivocerebellar and PC axons, it has been proposed that the rostral and caudal parts of the cerebellum have a mirror-image organization about the "rostrocaudal boundary" positioned on crus I (Sugihara and Shinoda, 2004; Sugihara and Quy, 2007; Sugihara et al., 2009). The present study has shown that the $\beta$-gal ${ }_{1 \mathrm{NM} 13}$-negative cluster is "split" into rostral and caudal clusters underneath the $\beta$-gal ${ }_{1 \mathrm{NM} 13}$-positive cluster in crus I. This would explain why there are similar projections outside of crus I and why crus I has a different axonal projection. We speculate a similar split of clusters might have also occurred in earlier embryonic date in some other clusters, thus underlying the general mirror-image organization. Overall, the present results indicate that crus I is the key lobule that determines the rostro-caudal arrangement of the cerebellum.

The nodulus (vermal lobule $\mathrm{X}$ ) and flocculus generally have their own clusters at E17.5. This indicates that these lobules are distinct divisions of the cerebellum from an early stage. On the other hand, the paraflocculus has a cluster organization similar to that in the lateral paramedian lobule, indicating that the paraflocculus could be regarded as a part of the caudal hemisphere (Sugihara et al., 2004).

\section{References}

Altman J, Bayer SA (1985) Embryonic development of the rat cerebellum. I. Delineation of the cerebellar primordium and early cell movements. J Comp Neurol 231:1-26. CrossRef Medline

Altman J, Bayer SA (1997) Development of the cerebellar system in relation to its evolution, structure, and functions. Boca Raton, FL: CRC.

Aoki E, Kimura R, Suzuki ST, Hirano S (2003) Distribution of OLprotocadherin protein in correlation with specific neural compartments and local circuits in the postnatal mouse brain. Neuroscience 117:593-614. CrossRef Medline

Arsénio-Nunes ML, Sotelo C (1985) Development of the spinocerebellar system in the postnatal rat. J Comp Neurol 237:291-306. CrossRef Medline

Ashwell KW, Zhang LL (1992) Ontogeny of afferents to the fetal rat cerebellum. Acta Anat (Basel) 145:17-23. CrossRef

Brochu G, Maler L, Hawkes R (1990) Zebrin II: a polypeptide antigen expressed selectively by Purkinje cells reveals compartments in rat and fish cerebellum. J Comp Neurol 291:538-552. CrossRef Medline

Dasen JS, De Camilli A, Wang B, Tucker PW, Jessell TM (2008) Hox repertoires for motor neuron diversity and connectivity gated by a single accessory factor, FoxP1. Cell 134:304-316. CrossRef Medline
Feirabend HK (1990) Development of longitudinal patterns in the cerebellum of the chicken (Gallus domesticus): a cytoarchitectural study on the genesis of cerebellar modules. Eur J Morphol 28:169-223. Medline

Flanagan JG, Vanderhaeghen P (1998) The ephrins and Eph receptors in neural development. Annu Rev Neurosci 21:309-345. CrossRef Medline

Fujita H, Sugihara I (2012) FoxP2 expression in the cerebellum and inferior olive: Development of the transverse stripe-shaped expression pattern in the mouse cerebellar cortex. J Comp Neurol 520:656-677. CrossRef Medline

Fujita H, Oh-Nishi A, Obayashi S, Sugihara I (2010) Organization of the marmoset cerebellum in three-dimensional space: lobulation, aldolase C compartmentalization and axonal projection. J Comp Neurol 518: 1764-1791. CrossRef Medline

Furutama D, Shimoda K, Yoshikawa S, Miyawaki A, Furuichi T, Mikoshiba K (1996) Functional expression of the type 1 inositol 1,4,5-trisphosphate receptor promoter-lacZ fusion genes in transgenic mice. J Neurochem 66:1793-1801. Medline

Furutama D, Morita N, Takano R, Sekine Y, Sadakata T, Shinoda Y, Hayashi K, Mishima Y, Mikoshiba K, Hawkes R, Furuichi T (2010) Expression of the IP3R1 promoter-driven nls-lacZ transgene in Purkinje cell parasagittal arrays of developing mouse cerebellum. J Neurosci Res 88:2810-2825. Medline

Goffinet AM (1983) The embryonic development of the cerebellum in normal and reeler mutant mice. Anat Embryol 168:73-86. CrossRef Medline

Hashimoto M, Mikoshiba K (2003) Mediolateral compartmentalization of the cerebellum is determined on the "birth date" of Purkinje cells. J Neurosci 23:11342-11351. Medline

Hashimoto M, Ito R, Kitamura N, Namba K, Hisano Y (2012) EphA4 controls the midline crossing and contralateral axonal projections of inferior olive neurons. J Comp Neurol 520:1702-1720. CrossRef Medline

Hirano S, Yan Q, Suzuki ST (1999) Expression of a novel protocadherin, OL-protocadherin, in a subset of functional systems of the developing mouse brain. J Neurosci 19:995-1005. Medline

Horn KM, Pong M, Gibson AR (2010) Functional relations of cerebellar modules of the cat. J Neurosci 30:9411-9423. Medline

Jessell TM, Sürmeli G, Kelly JS (2011) Motor neurons and the sense of place. Neuron 72:419-424. CrossRef Medline

Ji Z, Hawkes R (1995) Developing mossy fiber terminal fields in the rat cerebellar cortex may segregate because of Purkinje cell compartmentation and not competition. J Comp Neurol 359:197-212. CrossRef Medline

Karam SD, Burrows RC, Logan C, Koblar S, Pasquale EB, Bothwell M (2000) Eph receptors and ephrins in the developing chick cerebellum: relationship to sagittal patterning and granule cell migration. J Neurosci 20:6488 6500. Medline

Karam SD, Kim YS, Bothwell M (2001) Granule cells migrate within raphes in the developing cerebellum: an evolutionarily conserved morphogenic event. J Comp Neurol 12:127-135.

Korneliussen HK (1968) On the ontogenetic development of the cerebellum (nuclei, fissures, and cortex) of the rat, with special reference to regional variations in corticogenesis. J Hirnforsch 10:379-412. Medline

Larouche M, Che PM, Hawkes R (2006) Neurogranin expression identifies a novel array of Purkinje cell parasagittal stripes during mouse cerebellar development. J Comp Neurol 494:215-227. CrossRef Medline

Larsell O (1952) The morphogenesis and adult pattern of the lobules and fissures of the cerebellum of the white rat. J Comp Neurol 97:281-356. CrossRef Medline

Lin JC, Cepko CL (1998) Granule cell raphes and parasagittal domains of Purkinje cells: complementary patterns in the developing chick cerebellum. J Neurosci 18:9342-9353. Medline

Luckner R, Obst-Pernberg K, Hirano S, Suzuki ST, Redies C (2001) Granule cell raphes in the developing mouse cerebellum. Cell Tissue Res 303:159_ 172. CrossRef Medline

Marzban H, Chung S, Watanabe M, Hawkes R (2007) Phospholipase Cbeta4 expression reveals the continuity of cerebellar topography through development. J Comp Neurol 502:857-871. CrossRef Medline

Millen KJ, Hui CC, Joyner AL (1995) A role for En-2 and other murine homologues of Drosophila segment polarity genes in regulating positional information in the developing cerebellum. Development 121:3935-3945. Medline

Miyata T, Ono Y, Okamoto M, Masaoka M, Sakakibara A, Kawaguchi A, Hashimoto M, Ogawa M (2010) Migration, early axonogenesis, and 
Reelin-dependent layer-forming behavior of early/posterior-born Purkinje cells in the developing mouse lateral cerebellum. Neural Dev 5:23. CrossRef Medline

Nakagawa S, Watanabe M, Inoue Y (1997) Prominent expression of nuclear hormone receptor ROR alpha in Purkinje cells from early development. Neurosci Res 28:177-184. CrossRef Medline

Neudert F, Nuernberger KK, Redies C (2008) Comparative analysis of cadherin expression and connectivity patterns in the cerebellar system of ferret and mouse. J Comp Neurol 511:736-752. CrossRef Medline

Oberdick J, Schilling K, Smeyne RJ, Corbin JG, Bocchiaro C, Morgan JI (1993) Control of segment-like patterns of gene expression in the mouse cerebellum. Neuron 10:1007-1018. CrossRef Medline

Paradies MA, Eisenman LM (1993) Evidence of early topographic organization in the embryonic olivocerebellar projection: a model system for the study of pattern formation processes in the central nervous system. Dev Dyn 197:125-145. CrossRef Medline

Price SR, De Marco Garcia NV, Ranscht B, Jessell TM (2002) Regulation of motor neuron pool sorting by differential expression of type II cadherins. Cell 109:205-216. CrossRef Medline

Quy PN, Fujita H, Sakamoto Y, Na J, Sugihara I (2011) Projection patterns of single mossy fiber axons originating from the dorsal column nuclei mapped on the aldolase $\mathrm{C}$ compartments in the rat cerebellar cortex. J Comp Neurol 519:874-899. CrossRef Medline

Rakic P, Sidman RL (1970) Histogenesis of cortical layers in human cerebellum, particularly the lamina dissecans. J Comp Neurol 139:473-500. CrossRef Medline

Redies C (2000) Cadherins in the central nervous system. Prog Neurobiol 61:611-648. CrossRef Medline

Sgaier SK, Millet S, Villanueva MP, Berenshteyn F, Song C, Joyner AL (2005) Morphogenetic and cellular movements that shape the mouse cerebellum; insights from genetic fate mapping. Neuron 45:27-40. CrossRef Medline

Sillitoe RV, Hawkes R (2002) Whole-mount immunohistochemistry: a high-throughput screen for patterning defects in the mouse cerebellum. J Histochem Cytochem 50:235-244. CrossRef Medline

Sillitoe RV, Gopal N, Joyner AL (2009) Embryonic origins of ZebrinII parasagittal stripes and establishment of topographic Purkinje cell projections. Neuroscience 162:574-588. CrossRef Medline

Sillitoe RV, Vogel MW, Joyner AL (2010) Engrailed homeobox genes regulate establishment of the cerebellar afferent circuit map. J Neurosci 30: 10015-10024. CrossRef Medline

Smeyne RJ, Oberdick J, Schilling K, Berrebi AS, Mugnaini E, Morgan JI (1991) Dynamic organization of developing Purkinje cells revealed by transgene expression. Science 254:719-721. CrossRef Medline

Sotelo C (2004) Cellular and genetic regulation of the development of the cerebellar system. Prog Neurobiol 72:295-339. CrossRef Medline
Sotelo C, Bourrat F, Triller A (1984) Postnatal development of the inferior olivary complex in the rat. II. Topographic organization of the immature olivocerebellar projection. J Comp Neurol 222:177-199. CrossRef Medline

Sugihara I (2005) Microzonal projection and climbing fiber remodeling in single olivocerebellar axons of newborn rats at postnatal days 4-7. J Comp Neurol 487:93-106. CrossRef Medline

Sugihara I, Quy PN (2007) Identification of aldolase C compartments in the mouse cerebellar cortex by olivocerebellar labeling. J Comp Neurol 500: 1076-1092. CrossRef Medline

Sugihara I, Shinoda Y (2004) Molecular, topographic, and functional organization of the cerebellar cortex: a study with combined aldolase $\mathrm{C}$ and olivocerebellar labeling. J Neurosci 24:8771-8785. CrossRef Medline

Sugihara I, Shinoda Y (2007) Molecular, topographic, and functional organization of the cerebellar nuclei: analysis by three-dimensional mapping of the olivonuclear projection and aldolase C labeling. J Neurosci 27: 9696-9710. CrossRef Medline

Sugihara I, Ebata S, Shinoda Y (2004) Functional compartmentalization in the flocculus and the ventral dentate and dorsal group y nuclei: an analysis of single olivocerebellar axonal morphology. J Comp Neurol 470: 113-133. CrossRef Medline

Sugihara I, Fujita H, Na J, Quy PN, Li BY, Ikeda D (2009) Projection of reconstructed single Purkinje cell axons in relation to the cortical and nuclear aldolase C compartments of the rat cerebellum. J Comp Neurol 512:282-304. CrossRef Medline

Sürmeli G, Akay T, Ippolito GC, Tucker PW, Jessell TM (2011) Patterns of spinal sensory-motor connectivity prescribed by a dorsoventral positional template. Cell 147:653-665. CrossRef Medline

Voogd J, Pardoe J, Ruigrok TJ, Apps R (2003) The distribution of climbing and mossy fiber collateral branches from the copula pyramidis and the paramedian lobule: congruence of climbing fiber cortical zones and the pattern of zebrin banding within the rat cerebellum. J Neurosci 23:46454656. Medline

Wassef M, Sotelo C (1984) Asynchrony in the expression of guanosine $3^{\prime}: 5^{\prime}$-phosphate-dependent protein kinase by clusters of Purkinje cells during the perinatal development of rat cerebellum. Neuroscience 13:1217-1241. CrossRef Medline

Wassef M, Cholley B, Heizmann CW, Sotelo C (1992) Development of the olivocerebellar projection in the rat: II. Matching of the developmental compartmentations of the cerebellum and inferior olive through the projection map. J Comp Neurol 323:537-550. CrossRef Medline

Wilson SL, Kalinovsky A, Orvis GD, Joyner AL (2011) Spatially restricted and developmentally dynamic expression of engrailed genes in multiple cerebellar cell types. Cerebellum 10:356-372. CrossRef Medline 